\title{
TWO-FLUID SIMULATIONS OF GALAXY FORMATION
}

\author{
August E. Evrard \\ Physics Department, Univeristy of Michigan, Ann Arbor, MI 48103 \\ AND \\ F. J. Summers ${ }^{1}$ and Marc Davis \\ Astronomy Department, University of California, Berkeley, CA 94720 \\ Received 1993 January 7; accepted 1993 August 17
}

\begin{abstract}
We investigate the formation of galaxies and larger structure with a simulation modeling two gravitationally coupled fluids representing dark matter and baryons. The baryon gas dynamics are calculated with a smoothed particle hydrodynamics (SPH) method, and the physics modeled includes thermal pressure, shock heating, and radiative cooling. We simulate a $16 \mathrm{Mpc}$ periodic cube with $64^{3}$ particles in each fluid and $10 \%$ baryon mass fraction. The mass per baryon particle of $1.1 \times 10^{8} M_{\odot}$ implies an $L_{*}$ galaxy is resolved by $\sim 10^{3}$ particles, a factor 10 improvement over previous large-scale studies. Initial perturbations from a cold dark matter spectrum were generated such that a poor cluster of galaxies on a mass scale $10^{13.6} M_{\odot}$ would form. For computational reasons, the volume was evolved only to a final redshift $z=1$.

We identify a population of galaxy-like objects by searching for baryon concentrations above a fixed physical density equivalent to $10^{6}$ times the $z=0$ mean background value. These objects trace the overall filamentary structure in the dark matter at high $z$, and a subset drain into the cluster, which forms at the intersection of several filaments. With dissipation reducing their collision cross section, the simulated galaxies retain their identity within the cluster, alleviating the "overmerger problem" inherent in collisionless simulations of dark matter halos. The efficiency of galaxy formation is lower in the largest halos, as observed in rich clusters of galaxies.

The inferred abundance of galaxies grows rapidly to $z=2$ and flattens thereafter. The mass function has a low-mass slope shallower than that of the dark matter halos but steeper than the observed faint end slope of the luminosity function. Merger rates for galaxies are lower by a factor $\sim 2$ relative to dark matter halos.

We confirm, for the first time experimentally, disk formation as a natural consequence of hierarchical clustering in a large-scale cosmological environment. The majority of isolated galaxies exhibit centrifugally supported disks. A power-law relation between cold baryonic mass and maximum rotation velocity is found, $M \propto v_{\text {rot }}^{\alpha}$, with $\alpha=2.5$ after correcting for differential numerical resolution. The correlation is extremely tight, with rms residual equivalent to $0.15 \mathrm{mag}$. An assumption of $L \propto M^{p}$ with $p>1$ would both reconcile this relation with the observed Tully-Fisher law and produce a luminosity function faint end slope closer to that observed.

Both the spatial and velocity distributions of the simulated galaxies are biased with respect to the dark matter. The galaxy population is strongly biased at $z=3$, with spatial bias parameter $b_{\xi}=2$ or 3 for low- and high-mass samples. By $z=1$, the high-mass bias disappears and the low-mass sample is actually less clustered than the dark matter. A counts-in-cells analysis indicates that an unphysical degree of merging in the central cluster is likely responsible for the antibias signal in the correlation function. A robust, scale-dependent velocity bias is measured. The ratio of galaxy to dark matter pairwise velocity dispersions on a scale of $1 \mathrm{Mpc}$ is 0.7. The amplitude is only mildly dependent on redshift or mass cutoff and scales with separation as $r^{0.2}$.

The degree to which these results depend on numerical parameters is discussed. Mass resolution plays a key role in controlling the resulting fraction of cold, dense baryons. The mass fraction associated with galaxies decreases by a factor of $\gtrsim 3$ when the mass per particle is increased by a factor 8. Photoionization and energy input from supernova will have to be included to determine more carefully the fraction of highly dissipated material and the characteristics of the stellar component of galaxies.
\end{abstract}

Subject headings: galaxies: formation - hydrodynamics

\section{INTRODUCTION}

The formation of galaxies and large-scale structure is a fundamental problem of modern cosmology. The most likely scenario, strengthened by the recent results from the COBE DMR experiment (Smoot et al. 1992), is that structure formed via gravitational amplification of tiny perturbations in the density field imprinted at some early time after the initial hot, big bang.

\footnotetext{
${ }^{1}$ Present address: Princeton University Observatory, Peyton Hall, Princeton, NJ 08544.
}

In a universe dominated by nonbaryonic dark matter, galaxy formation involves both the formation of structure in the dark matter and the dissipative collapse of baryons into the dark matter potential wells (White \& Rees 1978). In this paper, we employ two-component numerical simulations designed to follow the gravitationally coupled evolution of dark matter and baryonic fluids to investigate the details of this process in a universe dominated by cold, dark matter (CDM).

The difficulties faced in attempting to model the galaxy formation process are severe. The dynamic range in all the prin- 
cipal physical quantities is large. For example, the optically bright regions of a galaxy are at a density contrast of $\sim 10^{6}$ above the cosmological background, while the cores of molecular clouds where star formation actually occurs are at least $10^{3}$ more dense than the galaxy on average. Simulations, by their nature, utilize discrete domains of space and time and I thereby impose limits on the dynamic range accessible to a given experiment. When attempting to squeeze the full problem into a finite computational domain, compromises are unavoidable. Furthermore, the modeling of known and unknown physics (e.g., shocks and star formation within a multiphase gas environment) introduces many tunable parameters. These extra degrees of freedom provide room for at least maneuvering toward, if not custom tailoring, a desired outcome.

These pitfalls have not discouraged a recent flurry of numerical attacks on the problem using combined $N$-body /three-dimensional gas dynamic algorithms explicitly designed for multicomponent systems. These efforts find roots in the early work of Larson $(1974,1975)$ who modeled the collapse of individual clouds comprised of gas and stars. More recent work extends the pioneering efforts of Carlberg (1988) and Carlberg \& Couchman (1989) who employed a so-called "sticky particle" method to model the baryonic component and locate sites for galaxy formation in a cosmology dominated by dark matter. Katz \& Gunn (1991) and Katz (1992) modeled the collapse of a single protogalactic cloud to investigate links between initial conditions and the star formation history and the final morphology of the collapsed object along the lines established by Larson. Navarro \& Benz (1991) reran the cosmological simulations of Zurek, Quinn and Salmon (1988) and pointed out the importance that feedback from star formation may play in producing centrifugally supported galactic disks. Umemeura (1992) has run simulations of pancake formation, focusing on the mass of fragments. Evrard \& Davis (1989) examined the fate of shock-heated baryons in a neutrino-dominated universe. Evrard (1990a, b) and Thomas \& Couchman (1992) investigated the problem of the dynamics of the intracluster medium in rich clusters of galaxies under a primordial infall hypothesis. Katz, Hernquist, \& Weinberg (1992) studied the evolution of baryons and dark matter in a random $22 \mathrm{Mpc}$ cubic region of a CDM universe.

Most of the studies cited above employed the Lagrangian technique of smoothed particle hydrodynamics (SPH) (Gingold \& Monaghan 1977; Lucy 1977) to model the baryonic fluid. A particular advantage of this approach is that it is highly adaptive-no spatial symmetries need be imposed to construct a solution. This capability is well matched to the problem of gravitational clustering from random initial density fields, since the latter generically do not have symmetries to exploit. The principal drawback of the scheme is that there is no firm framework for formal error analysis, primarily because of the lack of imposed geometry. The particles represent both the fluid and a deformable computational "grid." Checking the method for stability against variation of the numerical parameters has mostly been done empirically. However, the scheme has been benchmarked successfully against a number of representative problems with calculable solutions (see Monaghan 1992 for a recent review).

An alternative approach for modeling the gas is to use an Eulerian finite difference method such as those traditionally used in modeling astrophysical jets (e.g., Yuan, Centrella, \& Norman 1991) or aeronautical flows (Jameson 1989). Such approaches were used by Chiang, Ryu, \& Vishniac (1989), Ryu, Vishniac, \& Chiang (1990), Cen et al. (1990), and Cen \& Ostriker (1992b). These studies focused on determining the state of the intergalactic medium under the influence of shock heating and photoionization heating via bremsstrahlung emission from dense, hot plasma. At present, three-dimensional Eulerian codes are limited in studying galaxy formation by their fixed grid nature, which restricts the available dynamic range in density and mass. Adaptive, multigrid methods will ultimately be better suited for this problem.

When possible, we will attempt to compare our results with other published work. We caution that intercomparison of results from various codes and groups of workers is made complicated by a number of considerations. First, different scales may be probed by different experiments. Dynamic range limitations may leave no common ground between simulations probing disparate scales. For example, a simulation modeling the scale of rich clusters of galaxies $\left(10^{15} M_{\odot}\right)$ would provide no direct information on dwarf galaxies or Ly $\alpha$ clouds. Second, the input physics may differ between workers, making the conclusions from a comparison unclear. One must be careful to recognize which processes a simulation does or does not model. Third, approaches for implementing an unresolved element (e.g., feedback from star formation) may be distinctly different. The nonlinear (and chaotic) nature of the dynamics can readily amplify small differences in solutions into large ones. Fourth, widely varying methods for identifying a "galaxy" population are used. The statistics of each population may have important differences due to the characteristics of the defining algorithm. Finally, numerical issues, especially spatial and temporal resolution limitations, can play an important role in determining the character of a solution.

The paper is organized as follows. In $\S 2$, we present details of the simulation and present defining characteristics of the "galaxy" and "halo" populations. Section 3 provides results broken down into seven subsections addressing (i) the overall evolution of the spatial distribution, (ii) phase-filling factors, (iii) abundances, mass functions, and the relation between the simulated galaxies and halos, (iv) merger histories, (v) morphology, and (vi) correlation functions and biasing. The subsections are fairly independent so that a reader interested only in a specific topic can go directly to the relevant subsection without much penalty. A summary and discussion is given in $\S 4$. A Hubble constant $H_{0}=50 \mathrm{~km} \mathrm{~s}^{-1} \mathrm{Mpc}^{-1}$ is assumed throughout the paper.

\section{SIMULATION DESCRIPTION}

The aim of the simulation was to follow the dissipational collapse of galaxies along with their subsequent aggregation into a small cluster of galaxies. Resolving galaxies requires high spatial and mass resolution, while realistic treatment of a cluster requires including larger scale effects such as tidal torques and late infall. The former points toward treating small volumes while the latter favors large volumes. As a compromise value, we chose $16 \mathrm{Mpc}$ for the comoving box length in order to give higher weight to resolving galaxies. The mass per particle is such that an $L_{*}$ galaxy is resolved by roughly 1000 particles. With such resolution, one can model only a small cluster and not one as rich as Coma.

We model a periodic cube in a CDM universe with density parameter $\Omega=1$, baryon fraction $\Omega_{b}=0.1$, and present Hubble constant $H_{0}=50 \mathrm{~km} \mathrm{~s}^{-1} \mathrm{Mpc}^{-1}$. The spectrum was normalized such that the rms level of fluctuations within a 
fiducial $8 h^{-1} \mathrm{Mpc}$ sphere was $\sigma_{8}=0.6$. This corresponds to a bias parameter $b \equiv 1 / \sigma_{8}=1.7$. This normalization is appropriate to reproduce structure on the scale of clusters of galaxies, although it is $2.5 \sigma$ outside the range allowed by the $C O B E$ microwave background anisotropy measurements (White, Efstathiou, \& Frenk 1993). Possible solutions to this discrepancy involve adding extra physics to the "standard" CDM model; examples include a tilted primordial fluctuation spectrum, a nonzero contribution to the microwave fluctuations from gravity waves, or a combination of hot and cold dark matter (Efstathiou, Bond, \& White 1992).

The run contained 262,144 particles to represent each of the baryonic and dark matter components, for a total of 524,288 particles. Each baryon particle represents $1.08 \times 10^{8} M_{\odot}$ while each dark matter particle has mass $9.72 \times 10^{8} M_{\odot}$. The total mass in the simulation is $2.83 \times 10^{14} M_{\odot}$.

To ensure that a cluster formed in the simulated region, we generated initial conditions in a constrained manner using the technique developed by Bertschinger (1987). The algorithm generated a set of random amplitudes and phases drawn from the CDM spectrum subject to a constraint. The imposed constraint was that the density field smoothed with a Gaussian filter of radius $3.2 \mathrm{Mpc}$ about the center of the volume take on a value $3.6 /\left(1+z_{i}\right)$ at the initial redshift $z_{i}$. This constraint translates into a roughly $3 \sigma$ perturbations on the filter mass scale $3.6 \times 10^{13} M_{\odot}$. A DC component of the spectrum was permitted in order to mimic the effect of power on wavelengths larger than the simulated region. The amount of DC largescale power was sufficient to prevent us from evolving the region to the present day. Instead, we stopped the simulation at a redshift $z=1$ when the density parameter of the simulation was $\Omega=1.45$.

The initial displacement field generated above was normalized to a starting redshift $z_{i}=49$. We evolve the mass distribution to an intermediate redshift, $z=9$, by following a single, collisionless species using 500 steps and the expansion variable $a$ as the integration variable. At this point, a separate baryon particle distribution was introduced which traces the dark matter and is assumed isothermal at $T=10^{4} \mathrm{~K}$. The twocomponent system is then evolved to the final epoch with time as the integration variable and using 700 equal timesteps of $6.1 \times 10^{6} \mathrm{yr}$. The full evolution required $140 \mathrm{CPU} \mathrm{hr}$ on a Cray Y-MP supercomputer.

The starting redshift was chosen to ensure that all modes simulated were clearly in the linear regime. The $N$-body run from $z=49$ to $z=9$ follows the evolution of the growing mode perturbations until structure on the smallest resolvable scale, which we take to be a mass scale of 32 particles or $3.5 \times 10^{10} M_{\odot}$, is approaching nonlinearity. At $z=9$, there are a total of 10 dark matter halos with density contrast above 500 , which together contain $0.3 \%$ of the mass in the simulation. This mass fraction is small compared to the fraction of mass which ends up in galaxies (see $\S 3.2$ ). The intermediate redshift $z=9$ also corresponds to the epoch when the timescale for Compton cooling off the microwave background becomes larger than the expansion timescale, justifying a cold start for the gas. The initial temperature of $T=10^{4} \mathrm{~K}$ corresponds to the lowest temperature addressed by our cooling curve, but is arbitrary to some extent. As discussed below, choosing a lower temperature would have no effect on the dynamics, since $10^{4} \mathrm{~K}$ is already colder than the depth of any resolvable potential well at that epoch.

The simulation algorithm and benchmarking tests of it are described in Evrard (1988). P3MSPH is a combination of the P3M N-body code of Efstathiou \& Eastwood (1981) with SPH hydrodynamics as reviewed, for example, by Monaghan (1992). The code has been vectorized and runs at 90 mflops on a Cray Y-MP. Here we present only a brief outline of the pertinent details.

The baryonic density at the location of particle $i$ is calculated by convolving a weight, or smoothing function $W\left(r, h_{i}\right)$ over nearby particles

$$
\rho_{i}=m_{B} \sum_{j} W\left(\left|r_{j}-r_{i}\right|, h_{i}\right)
$$

where

$$
W\left(\left|r_{j}-r_{i}\right|, h_{i}\right)=\frac{1}{\pi^{3 / 2} h_{i}^{3}} e^{-\left|r_{j}-r_{i}\right|^{2} / h_{i}^{2}}
$$

is a Gaussian kernel with local smoothing scale $h_{i}$ and $m_{B}$ is the mass per baryon particle. The smoothing scale is allowed to vary both spatially and temporally to increase dynamic range. Information on the local entropy field is expressed in terms of a specific internal energy $\epsilon=k T / \mu \mathrm{m}_{p}(\gamma-1)$ where $\gamma=5 / 3$ is the assumed adiabatic index, $\mu$ is the mean molecular weight, and $m_{p}$ the proton mass. For simplicity, a mean molecular weight $\mu=0.6$, corresponding to an ionized gas of primordial composition, is assumed throughout the evolution and a minimum temperature of $10^{4} \mathrm{~K}$ imposed. The local pressure is described by an ideal gas equation of state

$$
P_{i}=(\gamma-1) \epsilon_{i} \rho_{i}
$$

Pressure gradients are measured via the gradient of the smoothing kernel. The gas force is written as an antisymmetric pairwise sum over neighboring particles to guarantee momentum and angular momentum conservation to machine accuracy

$$
\left(\frac{\nabla P}{\rho}\right)_{i}=m_{B} \sum_{j}\left(\frac{P_{i}}{\rho_{i}^{2}}+\frac{P_{j}}{\rho_{j}^{2}}\right) \nabla W\left(\left|r_{j}-r_{i}\right|, h_{i j}\right),
$$

where $h_{i j}=1 / 2\left(h_{i}+h_{j}\right)$.

A particle's thermal energy changes due to $P d V$ work, shock heating, and radiative cooling. Shock heating is incorporated using an artificial viscosity, which has the effect of increasing the pressure in supersonic, convergent flows from $P \propto c^{2}$ to $P \propto v^{2}$, where $c$ is the local sound speed and $v$ is the line of sight convergence velocity. Spherical cluster evolution (Evrard 1988; Hernquist \& Katz 1989) and planar shock tube experiments show that the form of the viscosity employed is effective in preventing interparticle penetration and satisfying shock jump conditions even in very strong shocks (Lattanzio et al. 1986). Radiative cooling rates for a gas of primordial composition are taken from Fall \& Rees (1985). The rates assume an optically thin plasma in collisional ionization equilibrium. Thermal bremsstrahlung, radiative recombination, dielectronic recombination, and excitation of discrete levels are included. Photoionization heating may be dynamically important in small halos, those with virial temperatures $T \lesssim 10^{5} \mathrm{~K}$ (J. Ostriker 1990, private communication; Efstathiou 1992). Since we cannot resolve halos in this temperature regime, we do not include photoionization processes explicitly. However, implicit in our choice of simulation parameters is the assumption that the baryons do not form structure below our mass resolution. Some form of stabilizing mechanism, possibly feedback from star formation, is necessary on these scales to prevent efficient 
cooling (Cole 1991; White \& Frenk 1991; Blanchard, VallsGabaud, \& Mamon 1992).

The gravitational force is softened by using the Plummer potential $\phi(r) \sim\left(r^{2}+\epsilon^{2}\right)^{-1 / 2}$ with $\epsilon$ fixed in the comoving frame. At $z=1$, the softening has the proper value $\epsilon=7 \mathrm{kpc}$. The same softening is used for gravitational interactions among gas-gas, gas-dark, and dark-dark particle pairs. The hydrodynamic smoothing length $h_{i}$ varies with local density $h_{i} \propto \rho_{i}^{-1 / 3}$ between a lower limit determined by either the Courant condition or $\epsilon / 2$ and an upper limit set by the size of the chaining mesh in the short-range force calculation. The Courant limit is dependent on the local temperature and shock activity, but does not pose the limiting constraint on the smoothing scale in the high-density regions we identify with galaxies. These regions have cooled to $10^{4} \mathrm{~K}$ and have $h_{i}=$ $\epsilon / 2 \simeq 4 \mathrm{kpc}$ at $z=1$. Changes in $h_{i}$ over one timestep are limited to $\delta h_{i} / h_{i}<5 \%$. We estimate that our spatial resolution in the densest regions is $\sim 13 \mathrm{kpc}$, which corresponds to roughly twice the gravitational softening and nearly 4 times the minimum hydrodynamic softening.

Table 1 summarizes the principal simulation parameters.

\subsection{Galaxy-like Objects}

What we call a "galaxy" in the simulation is a clump of cooled baryons with mean density higher than a fixed physical density equivalent to $\sim 10^{6}$ times the present mean background, corresponding to a particle density of about $0.5 \mathrm{~cm}^{-3}$ or a space density of $0.007 M_{\odot} \mathrm{pc}^{-3}$. This value is within a factor of a few of the baryon density contained within the inner $\sim 10 \mathrm{kpc}$ of bright galaxies. In practice, we employ a friends-offriends group finding algorithm which links together pairs of particles with separations less than $\eta_{g}(z)$ times the mean interparticle separation. We use

$$
\eta_{g}(z)=\eta_{1}\left(\frac{1+z}{2}\right)\left[\frac{\Omega(z)}{1.45}\right]^{1 / 3},
$$

where $\eta_{1}=0.02$ and $\Omega(1)=1.45$ are the values for the last simulation output.

Because we are dealing with high-density contrast objects, the friends-of-friends algorithm is sufficient to locate individual objects. Visual inspection of the output shows that the frequency of multiple objects is below $1 \%$. In contrast, when the method is applied at low overdensities $\sim 100$, extended bridges and tails can link together separate, unrelaxed halos. We use the term globs, for "galaxy-like objects," to describe the highdensity contrast baryonic clumps found in this manner. This is to avoid overuse (and blatant misuse) of the word "galaxy" to

TABLE 1

Summary of Model Parameters

\begin{tabular}{|c|c|}
\hline Parameter & Value \\
\hline Comoving length $L(\mathrm{Mpc})$ & 16 \\
\hline Total mass $\left(M_{\odot}\right)$ & $2.83 \times 10^{14}$ \\
\hline Total number of particles & 524,288 \\
\hline Gas particle mass $\left(M_{\odot}\right) \ldots \ldots \ldots$ & $1.08 \times 10^{8}$ \\
\hline Dark matter particle mass $\left(M_{\odot}\right)$ & $9.72 \times 10^{8}$ \\
\hline Gravitational softening $\epsilon$ at $z=1(\mathrm{kpc})$. & 6.8 \\
\hline Hydrodynamical resolution $h_{\min }(\mathrm{kpc})$.. & 3.4 \\
\hline Effective resolution $\sim 2 \epsilon \sim 4 h_{\min }(\mathrm{kpc}) .$. & 13 \\
\hline Timestep $\delta t(\mathrm{yr}) \ldots \ldots \ldots \ldots \ldots \ldots \ldots \ldots$ & $6.10 \times 10^{6}$ \\
\hline Initial redshift (DM only) . & 49 \\
\hline Initial redshift $(\mathrm{DM}+\mathrm{B}) \ldots$ & 9 \\
\hline 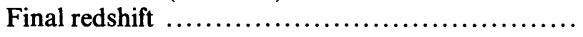 & 1 \\
\hline
\end{tabular}

represent lumps of imaginary particles in a computer. Despite our good intentions, we may occasionally slip in the term galaxy when discussing the simulated objects.

We feel that the galaxy objects we identify are the most reliable tracers of sites for galaxy formation to date (see also Katz et al. 1992 for similar results). Many methods of tracing galaxies in simulations have been implemented in the literature and most are physically plausible. However, one should recognize that if the tracing method is not solid, then the statistics of the galaxy tracer population are not robust. Too often one relies only on plausibility arguments and does not adequately test or explicitly specify the limitations of an algorithm. In that vein, an examination of the tracing properties of our method and several others appears in Summer, Davis, \& Evrard (1993). It should suprise no one that we find the above algorithm to be sound. Some limitations inherent in the simulation method are discussed below.

\subsection{Simulation Limitations}

Clearly, more physics is involved in forming a galaxy than gravity, pressure, shock heating, and radiative cooling. However, the elements included in this simulation are those classically thought to dominate the dynamics of bright galaxies (Rees \& Ostriker 1977; Silk 1977; White \& Rees 1978). Our attitude is that it is better to investigate individual physical elements one at a time rather than to attempt to include many coupled elements at once. The benefit of this approach is that it keeps the dynamical system relatively clean, with a minimum number of tunable parameters, and the major effects of each element may be identified. The obvious drawback is that certain physics is missing entirely and one must either justify its omission by showing the effects of its inclusion to be small or speculate as to the direction of the solution once the element is included.

To be specific, our simulation lacks star formation and therefore ignores associated feedback via supernova-driven winds and ionizing photons as well as the effect of metals on gas-cooling rates. The calculation presented by Dekel \& Silk (1986) demonstrated that supernova feedback is dynamically important in halos with a virial temperature below a critical temperature $T_{c}=2.5 \times 10^{5} \mathrm{~K}$. Similarly, photoionization from young stars or QSOs can heat the gas to temperatures near, but not above, the same critical temperature (Miralda-Escudé \& Ostriker 1989; Efstathiou 1992). The virial temperature of a collapsing dark matter halo can be estimated using a spherical model as

$$
T_{\text {vir }}=3.9 \times 10^{5}(1+z) M_{12}^{2 / 3} \mathrm{~K},
$$

with $M_{12}$ the mass in units of $10^{12} M_{\odot}$. The condition that a collapsed halo in the simulation have virial temperature above $T_{c}$ translates into a lower bound on $N_{p}$, the number of particles in the halo

$$
N_{p}>32\left(\frac{6}{1+z}\right)^{3 / 2} \text {. }
$$

Thus, all resolved ( $\gtrsim 32$ particles) halos collapsing earlier than $z=5$ will have virial temperature greater than $T_{c}$. However, baryons in small halos collapsing later than $z=5$ may be susceptible to disruption from photoionization and feedback. By $z=1$, halos just collapsed with fewer than 168 particles may have their cooling and star formation efficiencies strongly limited by these processes. On scales smaller than $\sim 10^{9} M_{\odot}$, 
the dynamical effects of photoionization heating are important (Cen \& Ostriker 1992a). This supports our implicit assumption that the baryons have not collapsed and cooled into smaller structures before the start of the calculation.

Presently, there is no unique prescription for determining star formation rates and associated feedback on galactic scales. Katz (1992) based his star formation rate on the local gas density, subject to a Jeans instability condition, and increased the thermal energy of gas in star-forming regions. However, the feedback energy was quickly radiated from the system and had little dynamical effect. Navarro \& White (1993) implemented a feedback scheme allowing for both thermal and kinetic energy input. A tunable parameter controlling the relative ratio of kinetic to thermal energy was found to strongly influence the density structure of the gas and hence determine the star formation rates and associated observables such as galaxy luminosity colors, and metal content.

Another consequence of not implementing a star formation algorithm is that the baryonic particles are treated as gaseous throughout. In particular, the effects of artificial viscosity may be overstated and the motions of the baryons in dense regions may be damped more than if they had become a collisionless stellar fluid. However, the dissipative collapse of the baryonic globs significantly reduces their cross-sectional area, making them less sensitive to artificial or thermal pressure forces. Visual inspection verifies that globs falling into the central cluster behave in a nearly ballistic manner within the hot, intracluster medium.

It is important to keep in mind the role of resolution limitations. There is a richness of structure and interesting physics operative on scales below our minimum resolved baryonic mass scale of $3 \times 10^{9} M_{\odot}$. Our simulation models the entire interstellar medium of our galaxy with $\sim 1000$ particles each as massive as 100 globular clusters. The hope is that this degree of resolution is sufficient to satisfy the primary aim of the simulation, which is to provide a more realistic manner of tracking the dynamical evolution of the galaxy population in a cosmological environment. The secondary motivation is to examine galaxy internal characteristics (morphological clues, for example), their temporal evolution, and dependence on environment. Future simulations with wider dynamic range will surely improve upon the results we present here.

\section{RESULTS}

\subsection{Spatial Structure}

Particle distributions in comoving slices of thickness $0.2 L=3.2 \mathrm{Mpc}$ are shown in Figures 1, 2, and 3 for $z=6.6$, 3.0 , and 1.0, respectively. Each figure is composed of two plots showing $(a)$ the entire simulation width and $(b)$ an enlargement of the central, boxed region of $(a)$ having comoving width $0.3 L=4.8 \mathrm{Mpc}$. The four panels within each plot show the dark matter distribution (upper left, labeled DM), the baryon distribution (upper right, B), dark matter particles in groups found with linking parameter $\eta=0.15$ (lower left, DM-G), and the baryons in groups identified with linking parameter $\eta_{g}(z)$ given in equation (5) (lower right, B-G). Not all particles are shown; particle counts are given in the figure captions. The DM-G panel is intended to highlight dark matter halos at a density contrast $\delta \sim 500$, slightly above the fiducial "virialized" value of 170 (Kaiser 1986). The B-G panel shows the glob population, i.e., likely sites for bright galaxy formation.
The frame at $z=6.6$ shown in Figure 1 is only $2 \times 10^{8} \mathrm{yr}$ after the starting redshift $z_{i}=9$ of the two-component part of the run. The large-scale mass distribution exhibits pancaking and filamentary structures which are generic to clustering from Gaussian random noise (Beacom et al. 1991; Kofman et al. 1992). There are 46 dark matter halos with 32 or more particles in the slice shown in Figure 1a. Their spatial distribution generally maps out the large-scale mass structures, but there are a few halos which appear in voids.

Only nine globs with 32 or more particles are found in the slice. The reason for the discrepancy in number compared to halos is that the baryons are still in the infall stage within their parent halos. The free-fall time from $100 \mathrm{kpc}$ is of order the age of the simulation for galactic-scale halos. The smaller linking parameter $\eta_{g}=0.068$ used for the baryons probes the inner regions of the dark matter halo where baryonic material has not yet had time to condense. For comparison, the group finder run on the dark matter distribution with smaller linking length $\eta=0.068$ finds only six groups with 32 or more members.

The situation is rather different at $z=3.0$ shown in Figure 2 . This frame is $1.3 \times 10^{9} \mathrm{yr}$ into the calculation. The obvious difference from Figure 1 is the more advanced state of the clustering. A central protocluster is evident, forming at the vertex of three filaments. There are 175 dark matter halos in the lower left panel of Figure $2 a$ and 102 baryon groups in the lower right panel. The spatial distribution of the latter on large scales mainly traces the caustic surfaces, with fairly empty voids occupying most of the slice volume. The largest dark matter halo has mass $M_{\mathrm{DM}}=1.4 \times 10^{13} M_{\odot}$ and contains 10 globs within a sphere defining a density contrast of 170 .

Examination of the subregion shown in Figure $2 b$ shows a good correspondence between dark matter halos and globs, particularly in the linear chain of objects extending diagonally away from the largest halo. The dark matter halos which do not show up in the baryon group plot ("empty" halos) tend to be low-mass systems near the 32 particle resolution limit. The internal dynamics of these systems is marginally resolved, so it not surprising to find that dissipation and collapse of their baryonic content has not occurred to the level required to satisfy the density and mass criteria imposed on the baryon groups. To satisfy the selection criteria, nearly all the baryons in the halo would have had to increase in density a factor greater than 66 above the mean halo density.

By the final epoch shown in Figure 3, the central cluster has grown to a mass of $M_{\mathrm{DM}}=5.5 \times 10^{13} M_{\odot}$ and now contains 26 globs within a sphere of overdensity 170 . There are 174 dark halos and 148 globs in the slice shown in Figure $3 a$. The overall distributions of dark matter and baryons shown in the upper panels are similar, the main difference being the rounder appearance of the shock-heated baryons in the central cluster, which results from the isotropic pressure tensor of the collisional intracluster medium. This feature has been documented in previous gas dynamic studies of clusters (Evrard 1990a, b; Thomas \& Couchman 1992).

\subsection{Filling Factors}

Since the gas in small halos has a cooling time that is shorter than both the local dynamical time and the Hubble time at any epoch of the simulation, one expects baryons in such systems to have cooled to the minimum imposed temperature of $10^{4} \mathrm{~K}$. Given infinite mass resolution and no sources of heating other than gravitational collapse, essentially all the baryons in the 


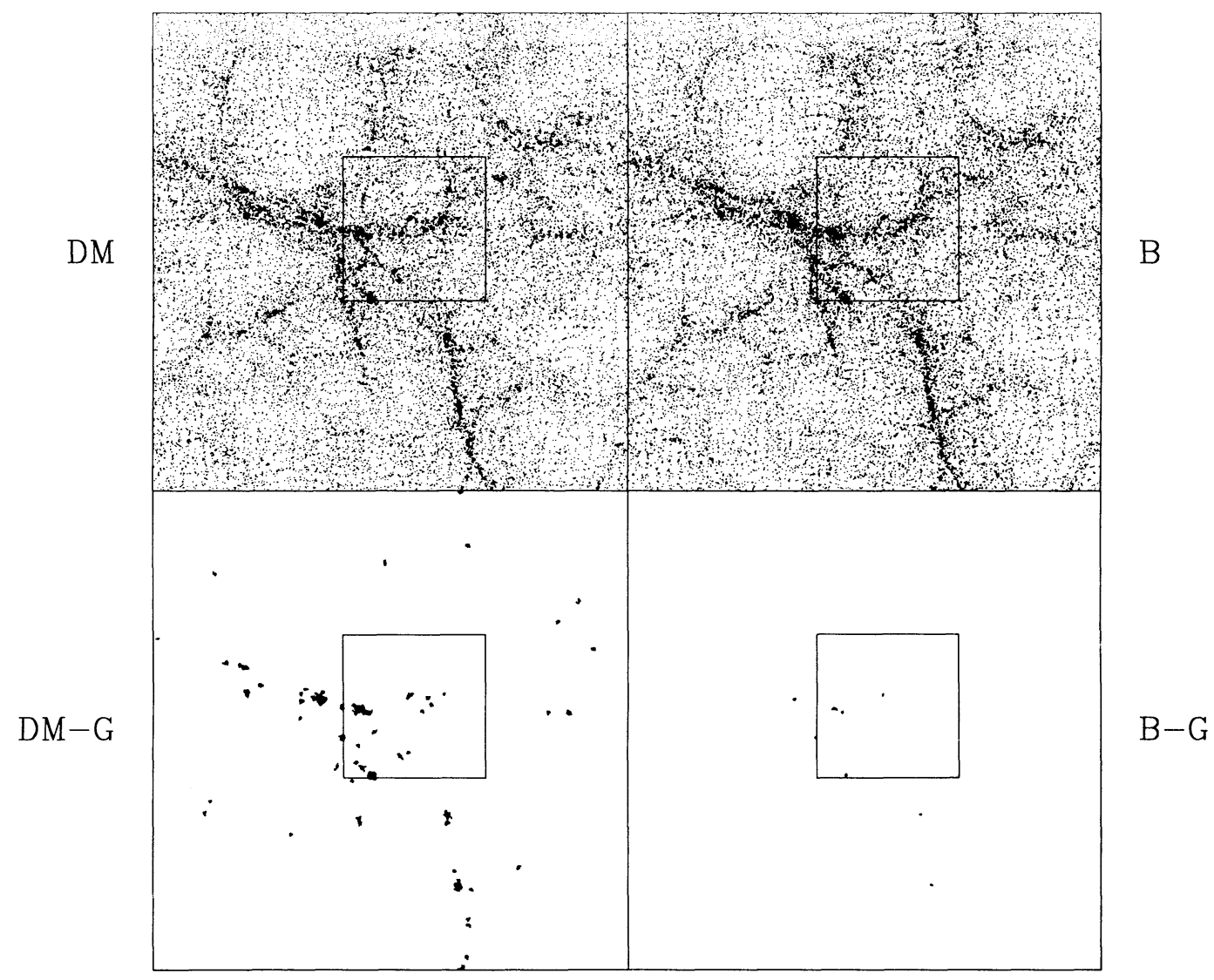

Fig. $1 a$

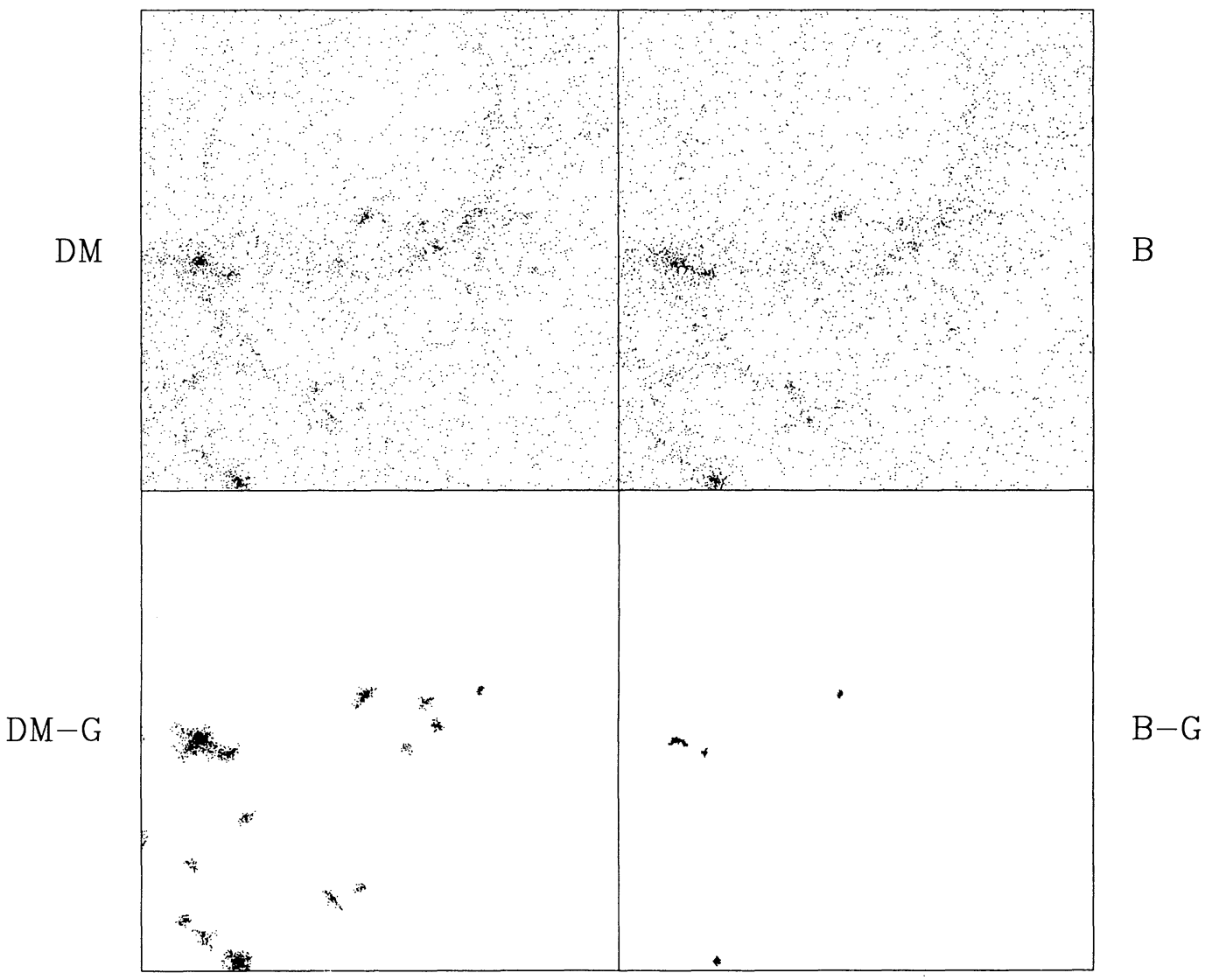

FIG. $1 b$

FIG. 1.-Projected particle distribution of a slice of thickness $0.2 L$ through the simulation at $z=6.6$. The quadrants show (clockwise from upper left) the dark matter particles (DM), baryonic particles (B), B particles in globs (B-G) and DM particles in halos (DM-G). For clarity, not all particles are shown. The numbers plotted/all particles in the full slice are: $30,051 / 85,470$ (DM); 29,968/85,446 (B); 743/743 (B-G); and 4895/4895 (DM-G). There are 46 DM halos and nine globs in the full slice. $(a)$ The full slice. $(b)$ Enlargement of the boxed region of length $0.3 L$ shown in $(a)$. 


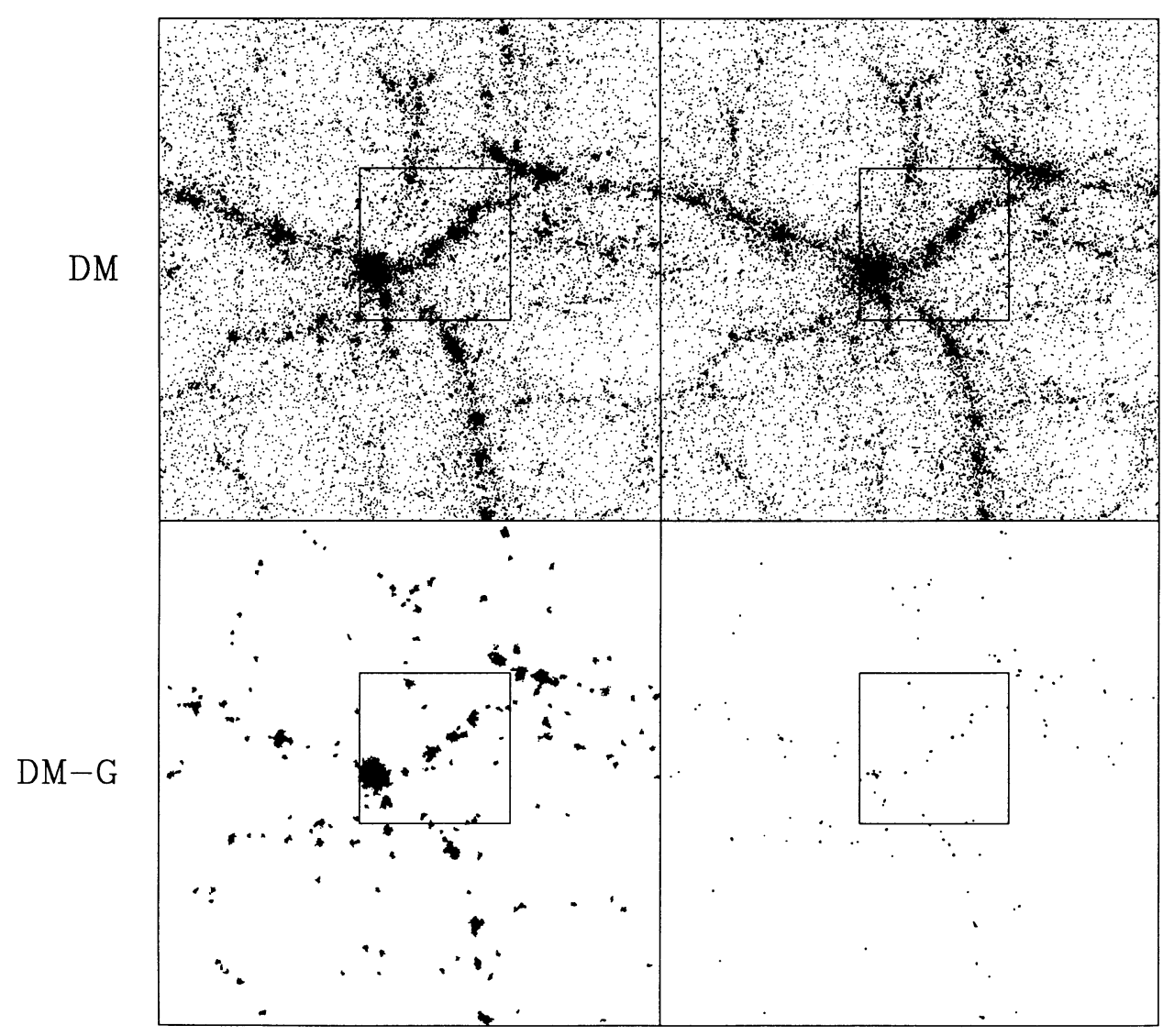

B

$B-G$

Fig. $2 a$

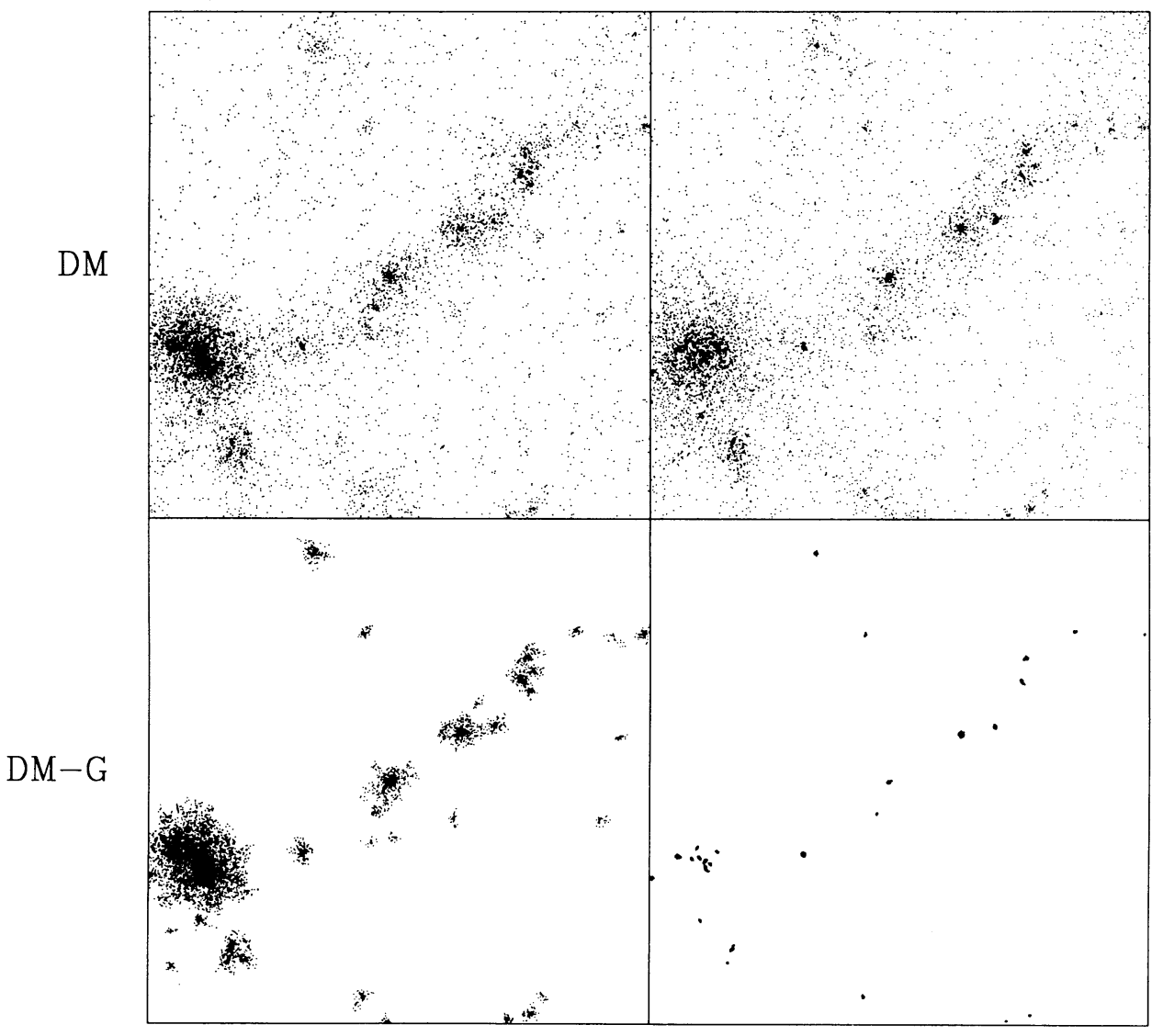

$\mathrm{B}$

$B-G$

FIG. $2 b$

Fig. 2.-Particle distributions at $z=3.0$. Format is the same as Fig. 1. The numbers plotted/all particles in the full slice are: 30,038/127,347 (DM); 30,019/127,227 (B); 17,760/17,760 (B-G); and 19,971/36,923 (DM-G). There are $175 \mathrm{DM}$ halos and 102 globs in the full slice. (a) Full slice. (b) Enlargement of boxed region in (a). 


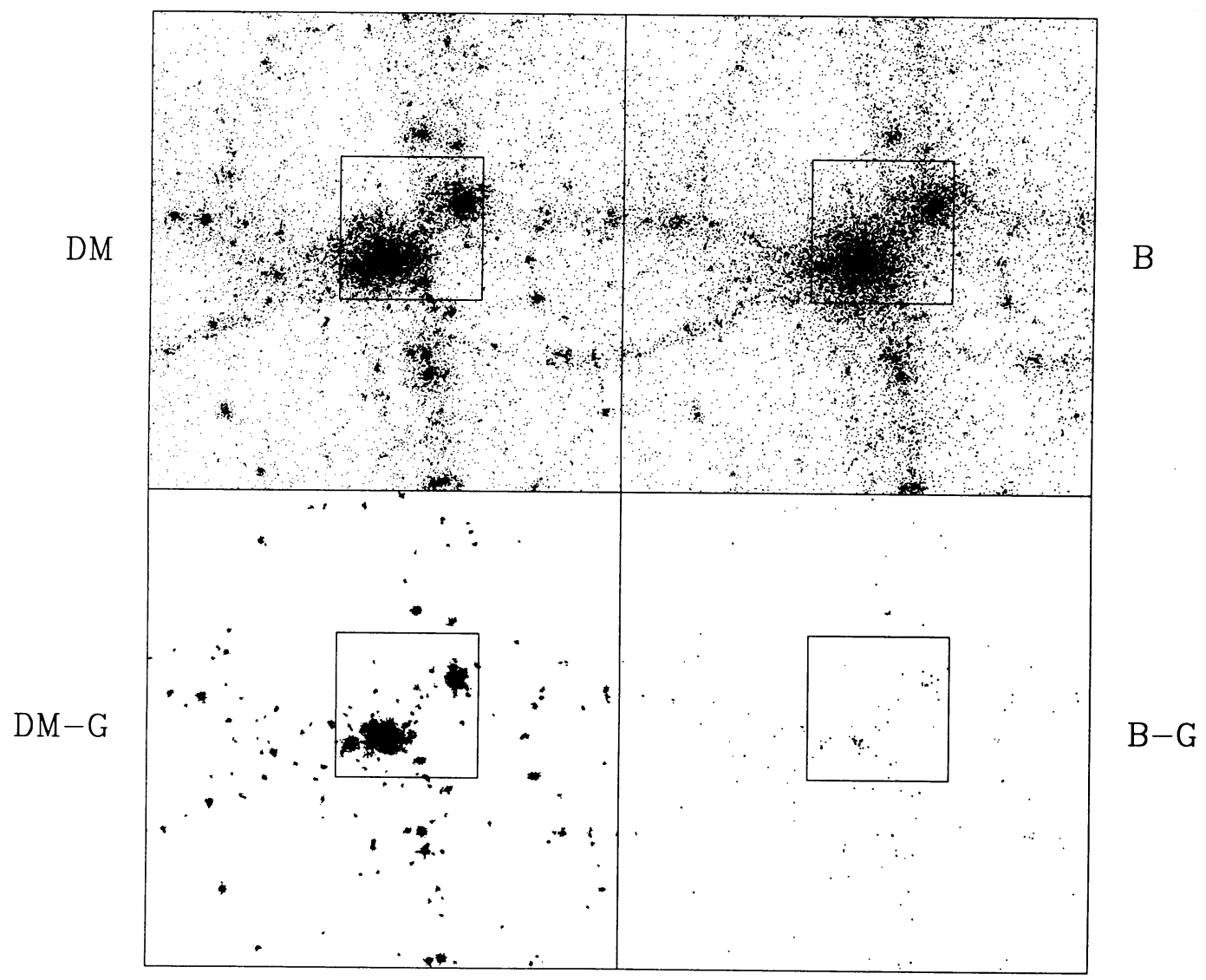

FIG. $3 a$

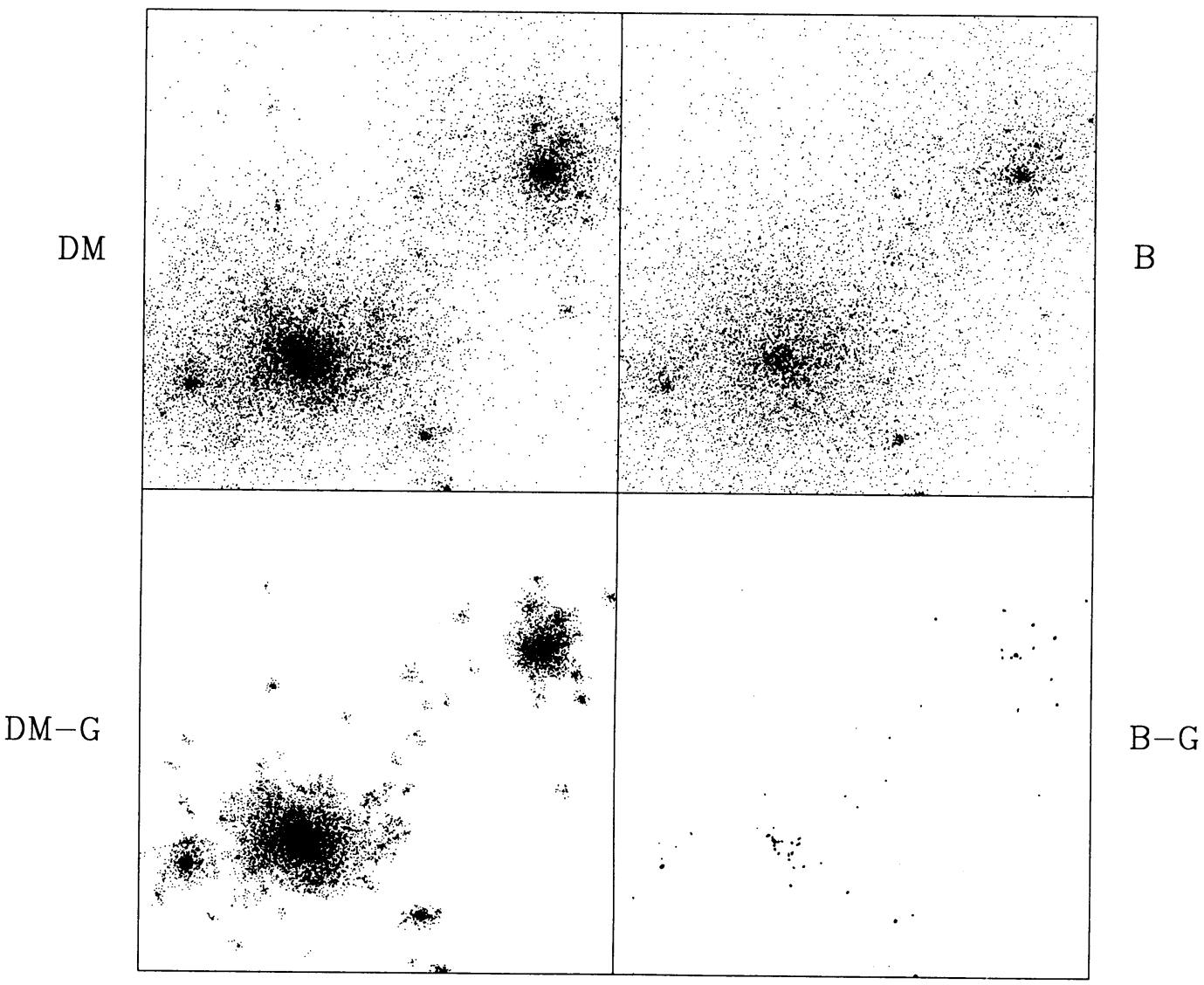

Fig. $3 b$

Fig. 3.-Particle distributions at $z=1.0$. Format is the same as Fig. 1. The numbers plotted/all particles in the full slice are: 29,898/179,523 (DM); $29,906 / 172,315$ (B); 19,987/40,385 (B-G); and 19,882/72,861 (DM-G). There are 174 DM halos and 148 globs in the full slice. (a) Full slice. (b) Enlargement of boxed region in $(a)$. 
universe would end up in a cold, dense phase (Cole 1991; White \& Frenk 1991; Blanchard et al. 1992). With some extra heating mechanism such as photoionization or supernova shocks, the cold, dense fraction can be reduced. In our simulation, the limited dynamic range prevents us from resolving cooling in halos containing less than $3 \times 10^{9} M_{\odot}$ in baryons.

One can probe the state of the particle fluids by examining the distribution in the density-temperature plane. Contours of the mass-filling factor $f_{m}(n, T) d \log n d \log T$, defined as the fraction of mass per unit logarithmic interval at density $n$ and temperature $T$, at $z=1$ are plotted in Figure 4. Following Faber's (1982) original convention, we plot the density axis inverted. In this way, the heavy dashed line, which divides the area into regions in which the cooling time is longer/shorter than the Hubble time, traces the shape of the input cooling curve (divided by one power of $T$ ).

For the dark matter, we use the one-dimensional peculiar velocity $v_{\text {pec }}$ to infer an effective "temperature" via

$$
T_{\mathrm{DM}}=\mu m_{p} v_{\mathrm{pec}}^{2} / 3 k,
$$

where $m_{p}$ is the proton mass, $k$ is the Boltzmann's constant, and a mean molecular weight $\mu=0.6$ is assumed. The factor of 3 corresponds to an assumption of $\beta=\sigma^{2} /\left(k T / \mu m_{p}\right)=1$ with $\sigma$ a one-dimensional velocity dispersion. The definition could be improved by separating the energy in bulk motion from the more truly "thermal" velocity dispersion, but the basic result would be unchanged. The dark matter contours show a single structure peaked at a temperature of a few million degrees and a density of $10^{-5} \mathrm{~cm}^{-3}$. Denser material tends to be hotter while the coolest mass is in the low-density voids. As expected, the minimum resolved mass scale imposes velocities with effective thermal temperature of $\sim 10^{5} \mathrm{~K}$ in collapsed regions.

In contrast, there are three distinct phases in the baryons: a hot, low-density phase, a cold, low-density phase, and a cold,

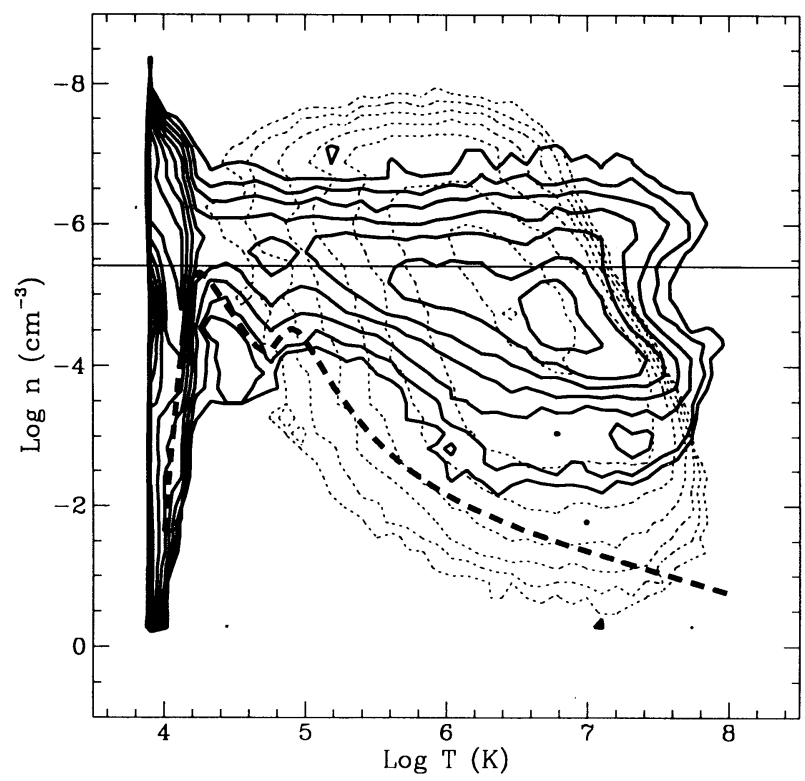

Fig. 4. - Contours of the mass weighted filling factor $f_{m}(n, T)$ in the densitytemperature phase space at $z=1$. Note the inverted density axis. The minimum contour is at $10^{-3.3}$ and contours are spaced logarithmically at intervals of 0.3 . The heavy, dashed line shows the locus of points for which the cooling time equals the final age of the simulation ( $4.7 \mathrm{Gyr}$ at $z=1)$. The solid contours show the baryon $f_{m}$, while the dashed contours show $f_{m}$ for the dark matter using the specific peculiar kinetic energy. high-density phase. The hot phase, peaked at $T=10^{7} \mathrm{~K}$ and $n=10^{-4.5} \mathrm{~cm}^{-3}$, represents both the intracluster medium in the central cluster and the intergalactic gas in smaller groups. The ridge extending toward $T=10^{6} \mathrm{~K}$ and $n=10^{-5} \mathrm{~cm}^{-3}$ arises from uncooled gas in the rarefied halos of individual galaxies. Note the contours trace out the cooling curve as expected. Baryons above the thick, dashed line have cooling times longer than the Hubble time at $z=1$ while those below the line cool rapidly to $10^{4} \mathrm{~K}$, making the cold, high-density ridge in Figure 4. Particles in this ridge are likely to be associated with galaxies in the simulation. The low-density, cold material corresponds to gas which has not collapsed into any resolved structures (i.e., in "voids"). Recall that with higher spatial and mass resolution, the fraction of cold gas in voids would decrease, either by being incorporated into small potential wells or by being transferred to the hot phase via feedback from star formation in these wells. Indeed, some dissipation or heating must occur if the model is to satisfy the Gunn-Peterson test.

These distinct phases are seen more clearly in Figure 5, where we plot the one-dimensional mass filling factor $f_{m}(n) d \log$ $n$ for hot $\left(T>10^{5} \mathrm{~K}\right)$ and cold $\left(T<10^{5} \mathrm{~K}\right)$ gas separately. The qualitative behavior of the results does not depend sensitively on the choice of temperature cut. The density structure of the hot phase is peaked just below $10^{-4} \mathrm{~cm}^{-3}$ and ranges from $10^{-6} \mathrm{~cm}^{-3}$ to $10^{-3} \mathrm{~cm}^{-3}$ with a tail extending to $10^{-2} \mathrm{~cm}^{-3}$. The cold phase is bimodal with a peak near the mean background density of $10^{-5.4} \mathrm{~cm}^{-3}$ and another peak at densities $\sim 1 \mathrm{~cm}^{-3}$.

The phase fractions are sensitive to the mass resolution of the experiment. We show in the figure results from a simulation which used 8 times fewer particles subsampled from the initial conditions of our larger run. The same three phases appear, but the mass fractions in the phases have changed substantially. Table 2 summarizes the fractions of baryons in each of the three principal phases. For the cold gas, the division between collapsed and void gas is imposed at a density contrast of 10. The principal effect of degrading the resolution is that mass is lost from the cold, collapsed phase and transferred into the hot phase. The cold, uncollapsed fraction remains nearly

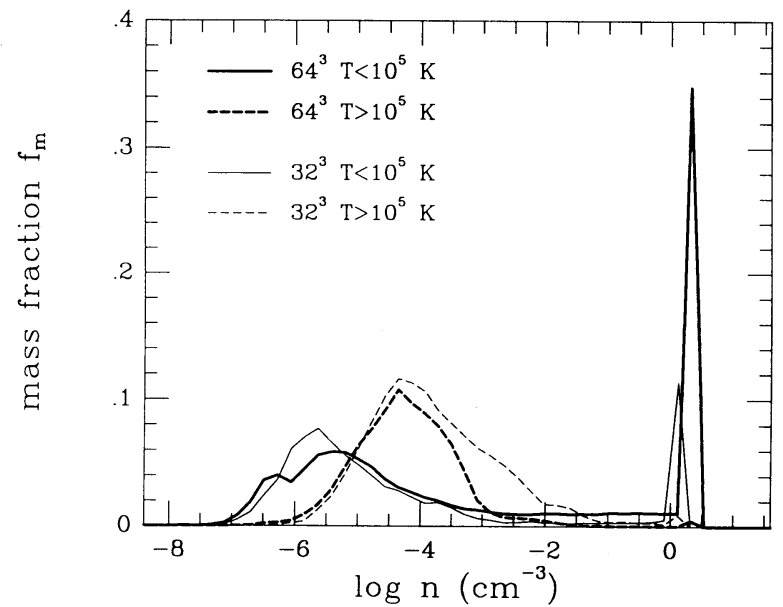

FIG. 5.-One-dimensional filling factors $f_{m}(n)$ for the cold and hot baryonic phases defined by a separation temperature of $10^{5} \mathrm{~K}$. Results are shown both for the $64^{3}$ particle run and a comparison run employing the same (subsampled) initial conditions with $32^{3}$ particles. The fraction of cold, dense baryons is strongly affected by mass resolution. 
TABLE 2

Mass Weighted Filling FaCtors

\begin{tabular}{|c|c|c|c|c|}
\hline \multirow[b]{2}{*}{ Phase } & \multicolumn{2}{|c|}{ P3MSPH } & \multicolumn{2}{|c|}{$\mathrm{C} \& \mathrm{O}$} \\
\hline & $32^{3}$ & $64^{3}$ & $32 \mathrm{Mpc}$ & $8 \mathrm{Mpc}$ \\
\hline Cold collapsed & 0.13 & 0.30 & 0.07 & 0.08 \\
\hline Hot $\ldots \ldots \ldots \ldots$ & 0.60 & 0.44 & 0.15 & 0.05 \\
\hline Cold void ..... & 0.27 & 0.26 & 0.78 & 0.86 \\
\hline "Galaxies" ............ & 0.05 & 0.18 & 0.07 & 0.08 \\
\hline
\end{tabular}

constant. The cause of the different behavior is straightforward. The minimum resolved mass scale in the $32^{3}$ run is 8 times larger than that in the $64^{3}$ experiment. As hierarchical clustering proceeds, gas collapsing into small ( $\sim 100$ particle) halos in the $64^{3}$ run will likely be resolved at a density high enough for the cooling time to be short. Such halos, represented by $\sim 15$ particles, are unresolved in the $32^{3}$ run and will have lower density estimates and longer cooling times. Subsequent collapse on a larger mass scale will push the gas to higher virial temperatures and lower average density. The result is that much of the material which would collapse and cool in the high-resolution experiment instead becomes part of the hot phase with long cooling time in the lower resolution experiment. Clearly, the mass fraction estimates in the various phases must be treated with caution.

Also in Table 2, we include the phase fractions from the Eulerian simulations of Cen \& Ostriker (1992a, hereafter C\&O) taken from the $z=1$ column of their Table 3. We quote the 32 and $8 \mathrm{Mpc}$ experiments $(h=0.5)$ which bracket the scale of our simulation. The defining criteria for their "galaxies" and "voids" categories match quite well with our "cold collapsed" and "cold void," respectively. We add their rows of "virialized gas" and "hot IGM" to compare to our "hot" phase factors. The former category could reasonably be ignored since it contains a maximum of $1 \%$ of the mass at $z=1$. There are significant differences between the phase fractions of $\mathrm{C} \& \mathrm{O}$ and our own. Around $80 \%$ of the mass in their simulations is in a cold void component $\left(T<10^{5} \mathrm{~K}, \rho / \bar{\rho}<10\right)$, while this fraction is near $25 \%$ in our experiments. The larger amount of cold, void material is compensated by correspondingly smaller amounts in the other two phases. Like ours, their higher resolution experiment, the $8 \mathrm{Mpc}$ run, shows a decreased fraction of hot material, but most of that fraction is transferred to the cold void component and not to the cold collapsed phase.

The principal difference in the input physics between our work and theirs is that $\mathrm{C} \& \mathrm{O}$ include photoionization heating and solve explicitly for the ionization state and cooling rate of the baryon plasma. They also assume a smaller value for the baryon fraction $\Omega_{b}=0.06$. The quandary is that both these differences should lead to a decreased amount of cooling relative to ours. The expectation is that their hot phase should be more prominent.

One explanation is that photoionization heating (which in their models is based on bremsstrahlung emission from the hot phase) is efficient at keeping the baryons suspended at $T \sim 10^{4}$ $\mathrm{K}$ and $\rho / \bar{\rho} \sim 1$, i.e., in the cold void phase. However, this is hard to understand since photoionization heating should not prevent the gas from falling into potential wells with virial temperatures $T_{\text {vir }}>10^{5} \mathrm{~K}$. At $z=1$, the spherical model, equation (6), implies that halos with mass $M_{h}>5 \times 10^{10} M_{\odot}$ should be hotter than $10^{5} \mathrm{~K}$. A simple estimate for the fraction of mass collapsed above that temperature is given by $f_{\text {coll }} \sim$ $\operatorname{erfc}\left[v_{c}\left(z, M_{h}\right)\right]$ with $v_{c}\left(z, M_{h}\right)=1.68(1+z) / \sigma_{0}\left(M_{h}\right)$ and $\sigma_{0}\left(M_{h}\right)$ the variance in the present, linear evolved density fluctuations smoothed on a mass scale $M_{h}$. For the parameters $h=0.5$ and $b=1.5$ employed by $\mathrm{C} \& \mathrm{O}$, we estimate $\sigma_{0}\left(5 \times 10^{10} M_{\odot}\right)=$ 10.5 which leads to $v_{c}=0.32$ at $z=1$ and, hence, $f_{\text {coll }} \sim 0.75$. A fraction of mass of this order should fall into these potential wells and show up either in the hot phase or in the cold collapsed phase, depending on the radiative cooling timescale. The sum of these two phases in our experiments is consistent with this calculation. We find $f_{\text {coll }}=0.73$ and 0.74 in the small and large runs, respectively. Conceivably, numerical diffusion or lack of resolution is responsible for keeping the baryons out of the deep potential wells in the C\&O models.

The fraction of the volume of space occupied by different phases $f_{v}(n, T) d \log n d \log T$ is presented in Figure 6. To construct the data for Figure 6, the density and mass weighted temperature at points on a $128^{3}$ cubic grid were determined by convolution of the particle information using the SPH smoothing kernel, equation (7), with individual particle smoothing lengths $h_{i}$. A lower limit on $h$ of $0.7 L / 128$ was imposed in order not to miss particles with smoothing lengths smaller than the grid spacing.

The phase structure in Figure 6 is markedly different from that seen in Figure 4. Most of the volume in the simulation contains cold, low-density material. The differences in phase structure between the dark matter and baryons are not as evident. The dynamic range in density is considerably reduced-density contrasts of a few times $10^{2}$ above or below the means background are resolved by a $128^{3}$ grid. There is good qualitative agreement between our filling factors and those of C\&O. In particular, both simulations show probability maxima at densities a factor $\sim 30$ below the mean and both exhibit a modest ridge in the baryon filling factor at $T=10^{4} \mathrm{~K}$ extending to high densities.

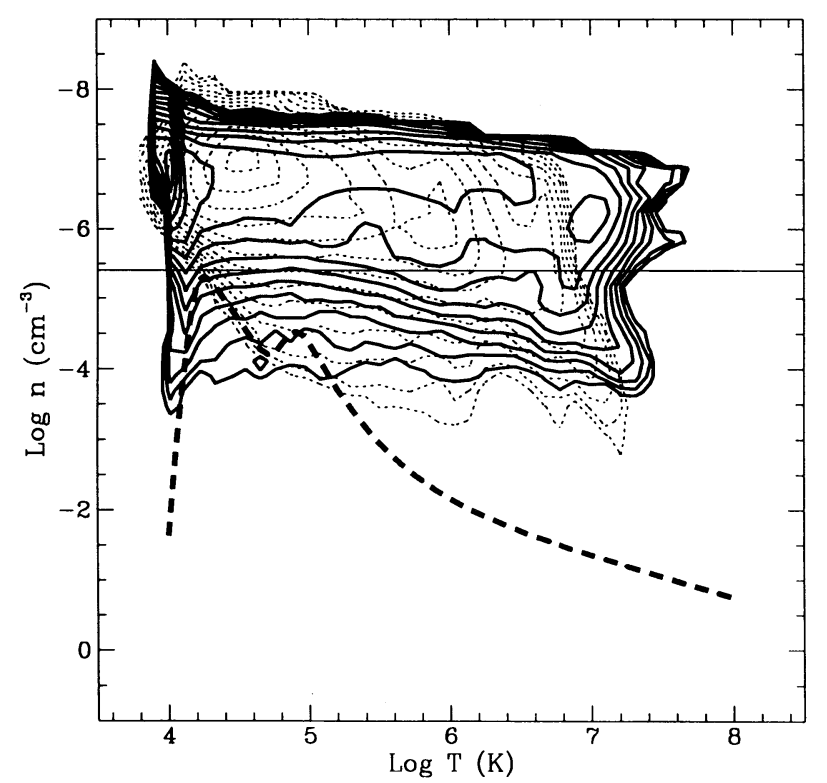

Fig. 6.-Contours of the volume weighted filling factor $f_{v}(n, T)$ at $z=1$ in cells of length $L / 128$. Format is identical to Fig. 4 . The minimum contour is at $10^{-4.2}$ and contours are spaced logarithmically by 0.3 . 


\subsection{Abundances, Mass Functions, and the Glob-Halo Connection}

In this subsection, we present results on the abundance and mass function of both halos and globs and discuss the relationship between the two populations.

Figure 7 shows the comoving abundance of globs and halos as a function of redshift. For globs, the number density of objects with masses above certain cutoffs is displayed with cutoffs corresponding to $32,128,512$, and 2048 particles, respectively. For halos, the number above 32 particles is shown. The curves have a shape characteristic of Gaussian random models (Cole \& Kaiser 1989; Cavaliere, Colafrancesco, \& Scaramella 1991). At high redshift, only rare, high overdensity regions have collapsed on a given mass scale $M$. The abundance of objects grows with decreasing redshift as objects form from the more numerous regions with lower initial overdensity. Finally, an epoch is reached when the overdensity required for collapse is below the rms value on that mass scale. The destruction rate of objects due to merging into larger mass scale systems begins to dominate the creation rate of new objects of mass $M$ and the abundance of objects begins to decline.

From Figure 7, it appears that the halos above the 32 particle mass limit are just beginning the merger-dominated phase. The number of globs above the lowest mass limit of $3.5 \times 10^{9}$ $M_{\odot}$ has remained fairly constant at a value of $0.05 \mathrm{Mpc}^{-3}$ between $z=2$ and $z=1$. As noted before, the more stringent criteria for identifying globs finds significantly fewer objects than are found for halos with similar particle counts. There exists a lag between halo formation and condensation of the baryons to galactic level. Because of the reduced merging of the globs (see $\S 3.4$ ), it would be interesting to see if further evolution of the glob number density follows the pattern established by the halos. The hierarchical nature of the formation process is evident in that the larger mass scales produce similar curves, but at later times.

There is a good deal of folklore regarding the claim that the CDM model "forms galaxies late" and therefore may be inconsistent with the existence of high-redshift QSOs (Turner 1991). We find the abundance of globs with baryon mass $M_{B}>1.4 \times 10^{10} M_{\odot}$ at $z=5$ is $\sim 0.002 \mathrm{Mpc}^{-3}$. This is comparable to the observed abundance of $L_{*}$ galaxies locally and

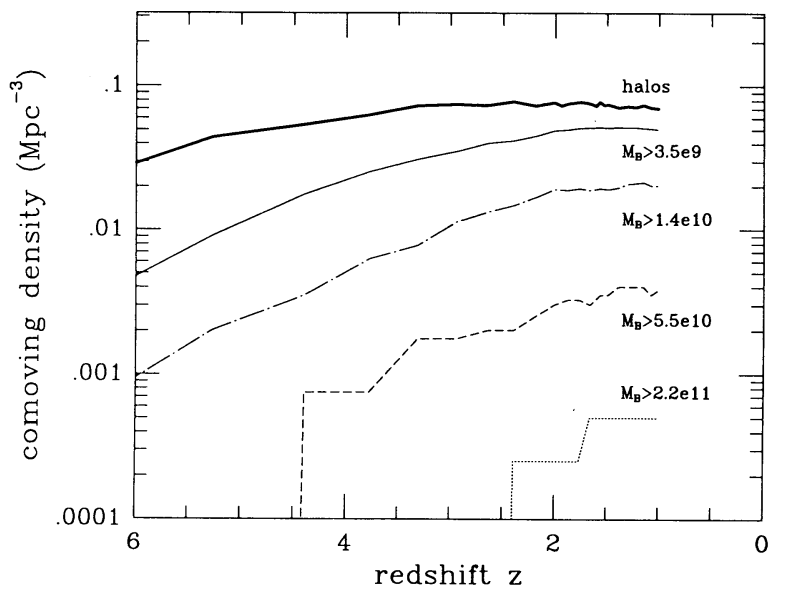

FIG. 7.-Number density of globs above indicated mass thresholds corresponding to $32,128,512$, and 2048 particles. The bold line give the number density of halos containing more than 32 particles. three orders of magnitude larger than the observed number density of QSOs at high redshift (Shanks et al. 1989). Even with considerable inefficiency in the formation mechanism of QSOs, it appears that the CDM model cannot be ruled out on the basis of the existence of QSOs at redshifts $z \gtrsim 5$. We caution that more specific statements are not possible without a detailed understanding of the conditions required for QSO formation (cf. Efstathiou \& Rees 1988). Also, the abundance of rare halos is exponentially sensitive to the product $(1+z)^{2} / \sigma_{8}^{2}$, with $\sigma_{8}$ the spectrum normalization parameter. Large $\sigma_{8}$ (low bias) models will produce a fixed abundance at a given mass scale earlier than high bias models.

The number and mass fraction of globs with 32 or more members at selected redshifts is displayed in Table 3. The latter is phrased in terms of a ratio to the critical density $\Omega_{\mathrm{gal}}$. At $z=1$, we find $\Omega_{\text {gal }} \simeq 0.018$, a value which is a factor $\sim 5$ larger than typical observational estimates (e.g., Persic \& Salucci 1992). Our value is somewhat larger than the value $\Omega_{\mathrm{gal}}=$ 0.013 quoted at $z=0.375$ in the CDM simulation of Katz et al. (1992). It is perhaps surprising that the two experiments yielded similar values for the fraction of baryons in galaxies. Katz et al. assumed $\Omega_{b}=0.05$ and their $32^{3}$ particle simulation in a slightly larger volume than ours led to a mass per baryonic particle of $1.2 \times 10^{9} M_{\odot}$, a factor of 10 larger than ours. Both of these factors should push in the direction of less efficient cooling and therefore a smaller fraction of mass in galaxies. On the other hand, Katz et al. evolved to a more recent redshift, thereby allowing a longer time to cool. Still, we infer $\Omega_{\text {gal }}=0.005$ from our $32^{3}$ particle simulation which has similar mass per baryonic particle as the Katz et al. study. The differences in detail between the two experiments make it difficult to say conclusively whether a significant discrepancy exists. The value of $\Omega_{\mathrm{gal}}=0.005$ inferred by $\mathrm{C} \& \mathrm{O}$ in their Eulerian experiments is similar to the result from our low-resolution run. Comparison between the two types of codes is very difficult. "Galaxies" in SPH codes are condensed groups of particles cooled to $10^{4} \mathrm{~K}$, while "galaxies" in the simulations of $\mathrm{C} \& \mathrm{O}$ were collections of cells tagged by a range of criteria such as local cooling time, divergence of the velocity field, and Jeans's stability.

The integrated mass function of globs and halos at redshifts $z=4.4,3.0,2.0$, and 1.0 is displayed in Figure 8. Bins a factor 2 wide in mass were used with the lower mass limit corresponding to 32 particles. The mass function of halos is nearly power law with differential slope close to -2 over most of the range. The expected exponential cutoff at high masses (Press \& Schechter 1974) is not apparent because of the constrained initial conditions which form a large central cluster. This cluster contains a cD-like object at its center which generates the high-mass extension of the glob mass function. In a large, unbiased region of space, objects such as this would be rare and the mass function would have the appropriate exponential cut off. The mass function of the halos changes little from $z=3$ to $z=1$, the exception being the growth of the large cluster.

TABLE 3

\begin{tabular}{crc}
\multicolumn{3}{c}{ BARYON FRACTION IN GLOBS } \\
\hline \hline$z$ & $N$ & $\Omega_{\text {gal }}$ \\
\hline $6.6 \ldots \ldots \ldots$ & 11 & 0.0003 \\
$4.4 \ldots \ldots \ldots$ & 70 & 0.0029 \\
$3.0 \ldots \ldots \ldots$ & 142 & 0.0080 \\
$2.0 \ldots \ldots \ldots$ & 205 & 0.0133 \\
$1.0 \ldots \ldots \ldots$ & 203 & 0.0183 \\
\hline
\end{tabular}




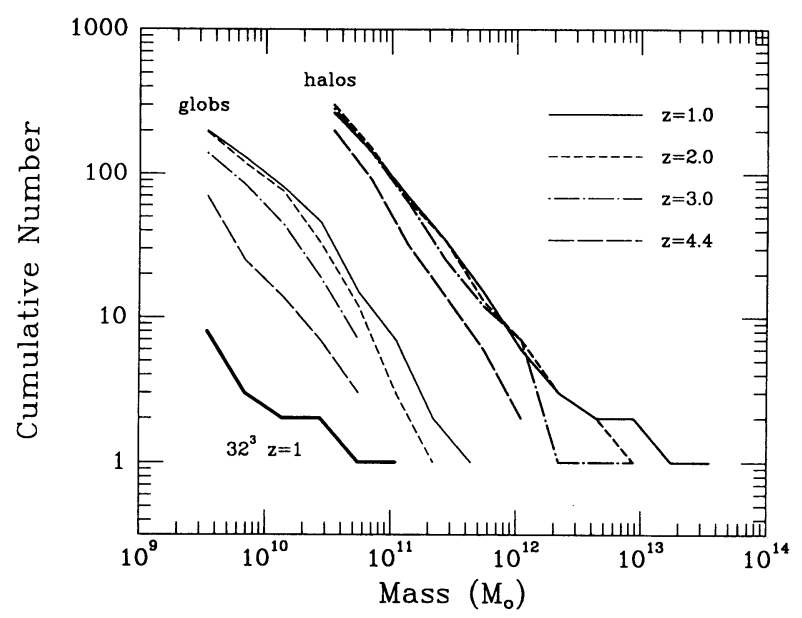

Fig. 8.-Cumulative mass function of globs and halos at various redshifts. Also shown is the glob mass function from the $32^{3}$ particle run at $z=1$.

The mass function of globs, in contrast, evolves noticeably from $z=3$ to $z=1$ and is not well described by a power law at late times. Pure power-law fits to the data at $z=2$ and 1 yield $\chi^{2}$ values of 21.3 and 22.8 for 6 and 7 degrees of freedom, respectively. Fits of the differential mass functions (shown in integral form in Fig. 8) to a Schechter form

$$
\Phi(M) d M=\Phi_{*}(z)\left[M / M_{*}(z)\right]^{\alpha_{g}(z)} \exp \left(-M / M_{*}\right) d M / M_{*}
$$

are given in Table 4 for $z=3,2$, and 1. Errors were determined assuming Poisson statistics for the number of objects in each mass bin. The high-mass cutoff in the glob mass function arises from the inability of the baryons in the largest collapsed halos to cool efficiently. The reason for the change in low-mass slope relative to the halos is less obvious. It arises partly from a pileup in the number of objects around $M_{*}$ (due to the truncation of the merger hierarchy) and partly from a decrease in the number of low-mass objects due to less efficient dissipation.

The differential mass function for the globs at $z=1$ is plotted in Figure 9 along with the locally determined luminosity function from Loveday et al. (1992) scaled assuming a baryon mass-to-light ratio of $5(h=0.5)$. The low-mass slope in the experiment is steeper than the faint end slope of the luminosity function $\alpha_{L}=-0.97 \pm 0.015$ observed in the Stromlo-APM redshift data. That value of $\alpha_{L}$ is somewhat flatter than the canonical $\alpha=-1.25$ derived from previous redshift surveys (Sandage, Tammann, \& Yahil 1979; Kirshner et al. 1983). Observational complications due to the uncertain numbers of dwarf and low surface brightness galaxies (Phillipps \& Disney 1985 ) might be a serious problem for the local studies. Deep surveys of the Virgo cluster indicate a nearly exponential rise in the number of faint dwarf elliptical galaxies (Bingelli, Sandage, \& Tammann 1985). Bothun, Impey, \& Malin (1991) suggest the faint end slope may be closer to $\alpha_{L} \simeq-1.6$ when corrections for low surface brightness galaxies are included.

TABLE 4

Schechter Fits to Glob Mass Function

\begin{tabular}{cccccc}
\hline \hline$z$ & $\Phi_{*}\left(\mathrm{Mpc}^{-3}\right)$ & $\alpha_{g}$ & $M_{*}\left(10^{10} M_{\odot}\right)$ & $\chi^{2}$ & $N_{d f}$ \\
\hline $3.0 \ldots \ldots \ldots$ & $0.0052 \pm 0.0031$ & $-1.49 \pm 0.21$ & $6.2 \pm 5.5$ & 0.7 & 2 \\
$2.0 \ldots \ldots \ldots$ & $0.0094 \pm 0.0032$ & $-1.39 \pm 0.14$ & $5.3 \pm 1.8$ & 7.2 & 5 \\
$1.0 \ldots \ldots \ldots$ & $0.0080 \pm 0.0026$ & $-1.39 \pm 0.12$ & $7.2 \pm 2.7$ & 7.7 & 6 \\
\hline
\end{tabular}

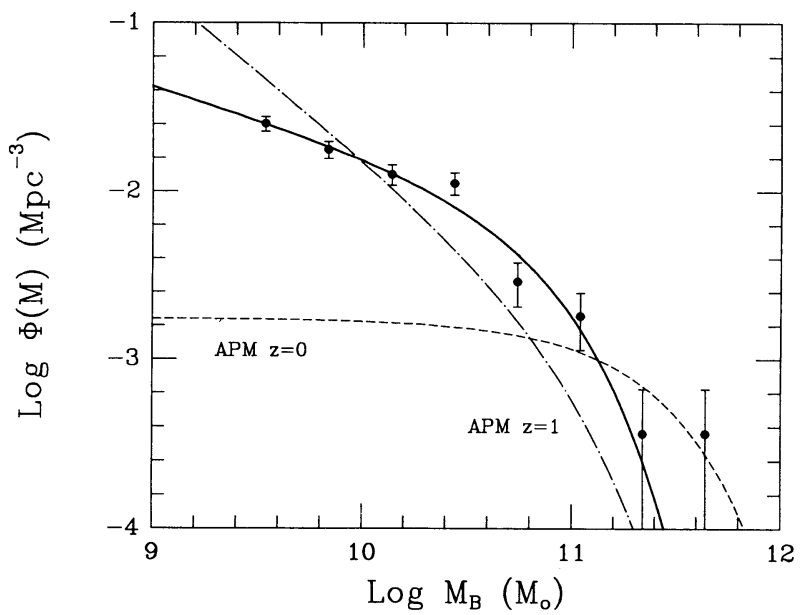

Fig. 9.-Differential mass function for the globs at $z=1$ (dots) with Poisson error bars and the best-fit Schechter function (solid line) with parameters given in Table 4. The dashed line is the APM luminosity function scaled to mass assuming a baryon $M / L=5$. The dot-dashed line shows the luminosity function inferred at $z=1$ from the toy model of Maddox et al. (1991) fitted to the APM counts.

Our steep, faint end slope is expected from analytic considerations such as a Press-Schechter analysis, but bear in mind that it is a slope of a mass function, not a luminosity function.

A more serious discrepancy than the shape of the abundance function is the overall normalization. The number of globs produced at moderate to low masses far exceeds the number of local galaxies in the Stromlo-APM and other surveys. (The effect of the central cluster itself on the number density of objects is not extreme since only $\sim 15 \%$ of the globs produced are contained in the cluster.) It is not entirely clear whether this is a problem, since the simulation results correspond to the epoch $z=1$ whereas the luminosity function is only known at $z=0$. Analyses of faint galaxy counts in the blue suggest a significant evolution of the luminosity function at modest lookback times (Broadhurst, Ellis, \& Shanks 1988; Colless et al. 1989; Lilly, Cowie, \& Gardner 1991). To illustrate the potential scale of the required evolution, we plot in Figure 9 the luminosity function at $z=1$ expected from the evolution model of Maddox et al. (1990). Their model employed a Schechter function with $\alpha_{L}$ and $L_{*}$ varying with redshift in such a way as to reproduce the low-redshift $(z \simeq 0.1-0.2)$ blue galaxy counts in the APM survey. An order of magnitude increase in the abundance of $L_{*} / 10$ galaxies is not out of the question. The models examined by Lilly et al. (1991) require an increase in $\Phi_{*}$ of roughly a factor 2.5 by a redshift $z=0.4$. Such strong evolution would require a complex star formation history along the lines suggested by Broadhurst et al. (1988) to fit both the $\mathrm{K}$ and $B$ band counts.

To illustrate the range of galactic environments accessible within the simulation as well as to examine the effect of environment on galaxy mass, we plot in Figure 10 the glob mass against the local galaxy density $\Delta_{g}$. The latter is estimated using the distance to the $n$th nearest glob $r_{n}$ and the unbiased estimator (Casertano \& Hut 1985)

$$
\Delta_{g}=\frac{V}{N} \frac{n-1}{V\left(r_{n}\right)},
$$

where $N$ is the total number of objects in the volume $V$ and $V\left(r_{n}\right)$ is the volume of the sphere which just touches the $n$th 


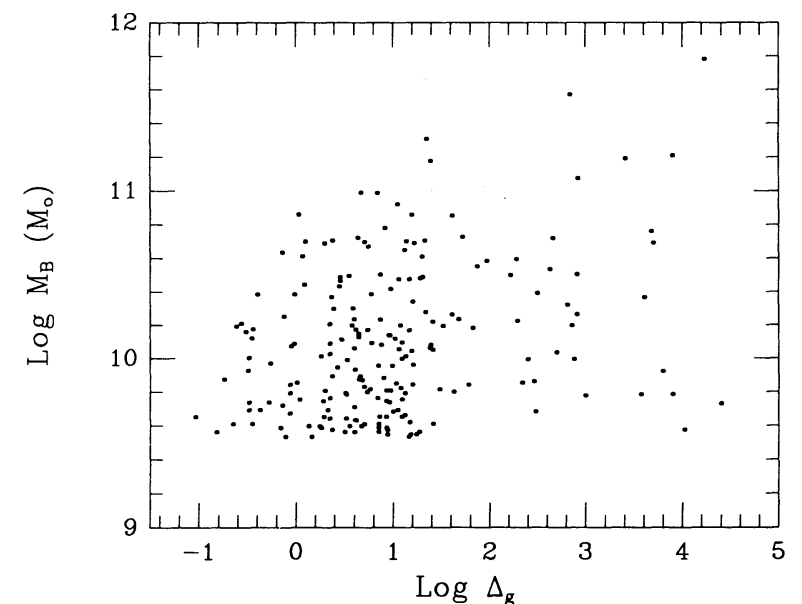

Fig. 10.-Glob mass vs. local density based on the distance to the sixth nearest galaxy at $z=1.0$.

neighbor. We use $n=6$. The dynamic range in $\Delta_{g}$ is over five orders of magnitude, from $10^{-1}$ to $10^{4}$. Nearly all the globs with $\Delta_{g}>10^{2}$ are members of the central cluster. There is a trend for the median density to increase with increasing glob mass. The most massive systems are likely to be found in cluster environments while smaller objects tend to populate the "field." However, at any given galaxy density, a range of glob masses is evident.

One can get a clearer idea of the relationship between globs and halos by looking at direct associations between objects of each class. The top part of Figure 11 shows the glob occupation number $N_{\text {glob }}$, defined as the number of globs within a radius $R_{500}$ of each dark matter halo. Note that the physical radius at $z=1$ scales with halo mass as

$$
R_{500}=100\left(M_{\mathrm{DM}} / 10^{12} M_{\odot}\right)^{1 / 3} \mathrm{kpc} .
$$

The 246 halos span 3.5 decades in mass. The large cluster has a mass of $3.5 \times 10^{13} M_{\odot}$ and contains 19 globs within $R_{500}$. Extending to a density contrast of 170 , we find 26 globs within a radius $R_{170}=533 \mathrm{kpc}$. The second largest system, containing 10 globs, is located $1.1 \mathrm{Mpc}$ from the largest cluster and is infalling with a relative velocity of $400 \mathrm{~km} \mathrm{~s}^{-1}$. The two will merge in $\sim 2$ Gyr. After these two halos, there are 12 other halos containing more than one glob. A further 135 halos each contain a single glob. (These systems will hereafter be referred to as isolated.) The 97 halos with $N_{\text {glob }}=0$ either contain "protoglobs" (71) or have had their gas content modified by the cluster environment (26).

In our simulation, all halos with $M_{\mathrm{DM}}>10^{12} M_{\odot}$ are multiple systems. Halos in the range $2.5 \times 10^{11} M_{\odot}<M_{\mathrm{DM}}<10^{12}$ $M_{\odot}$ may be either isolated or multiple. Below this range, down to about $10^{11} M_{\odot}$, one finds mostly isolated halos. At smaller halo masses, the time lag between halo formation and glob formation introduces considerable uncertainty. The naive assumption of a one-to-one correspondence between halos and galaxies is a gross simplification, but we do not have enough statistics to be able to quantify the trend of glob occupation number with halo mass.

The lower portion of Figure 11 shows the masses of the individual globs identified with each halo plotted in the upper part. Isolated globs are plotted as points while circles are used for globs in multiple systems. The solid line is the baryonic mass associated with the halo mass under our assumed $\Omega_{b}$. For

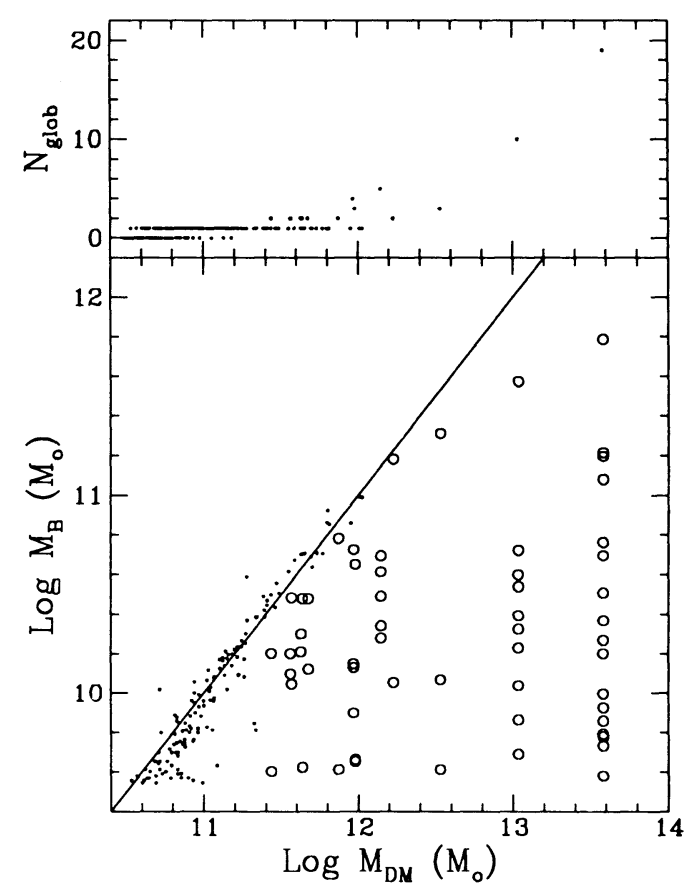

FIG. 11.-Halo occupation number $N_{\text {glob }}$ and glob mass within each halo as a function of halo mass. Circles in the lower panel indicate halos containing multiple globs. The line in the lower panel is $M_{B}=\Omega_{b} M_{\mathrm{DM}}$.

the multiple systems, the mass of the largest glob increases roughly as $M_{\mathrm{DM}}^{0.4}$. The large multiple systems show a range of masses down to our resolution limit, with a relatively large mass ratio between the first and second ranked globs. Above $M_{\mathrm{DM}}=10^{11} M_{\odot}$, the glob masses in the isolated systems agree well with the baryonic equivalent of the dark halo mass. For smaller halos, the glob mass falls somewhat short of $\Omega_{b} M_{\mathrm{DM}}$. This is most likely due to lack of resolution; these halos are comprised of fewer than 100 particles.

These trends are apparent in Figure 12, where we plot the "galaxy formation efficiency" in dark matter halos. Here the total baryonic mass of globs in a halo, $M_{B . t o t}$, is used to compute an efficiency $\epsilon_{g}$ as the ratio

$$
\epsilon_{g}=\frac{M_{B, \text { tot }}}{\Omega_{b} M_{\mathrm{DM}}} .
$$

This ratio should be unity if all the baryonic mass initially

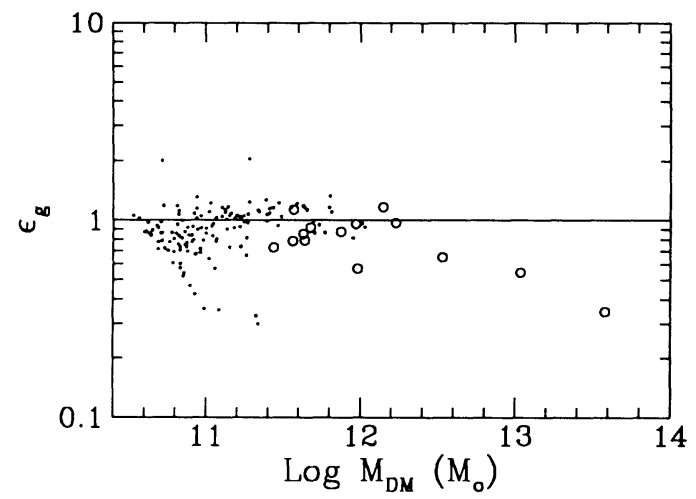

FIG. 12.-Galaxy formation efficiency $\epsilon_{g}$ vs. halo mass. Circles indicate halos with multiple globs. 
associated with the halo mass within $R_{500}$ can cool and condense to the center of the halo. Multiple systems are represented by circles. For both isolated and multiple halos with $M_{\mathrm{DM}} \lesssim 10^{12} M_{\odot}, \epsilon_{g}$ scatters about unity. For the three halos above this mass scale, $\epsilon_{g}$ decreases monotonically with $M_{\mathrm{DM}}$, falling to $\frac{1}{3}$ for the large, central cluster.

This behavior is consistent with that anticipated from timescale arguments (White \& Rees 1978). In the shallow and relatively quiescent potential wells of isolated galaxies, the gas is able to cool and sink to the center over a Hubble time, if need be. In a cluster environment, a galactic scale potential well has a shorter amount of time to accrete cooling material before undergoing a close encounter or merger as the clustering hierarchy builds. Mechanisms such as shock heating, tidal stripping, or ram pressure stripping can remove a reservoir of galactic gas or prevent it from dissipating into the local galactic potential (Larson, Tinsley, \& Caldwell 1980). Instead the gas winds up suspended in the larger scale potential well as the tenuous, intracluster medium (ICM) seen in X-rays (Sarazin 1986).

Mass estimates based on observations of the ICM in clusters indicate a trend with cluster richness in the ratio of ICM mass to stellar mass in galaxies (David et al. 1990; Arnaud et al. 1991). Hotter clusters have a fractionally higher mass in the ICM, indicating less efficient galaxy formation in more massive clusters, consistent with the results in Figure 12.

\subsection{Merger History}

An important characteristic of hierarchical structure formation scenarios is the ubiquity of merging. Collisionless simulations have shown that merging efficiently erases substructure such that the mass distribution of collapsed objects is smooth at density contrasts above $\sim 500$. The compact globs should be relatively free from this "overmerging" problem. We now examine this issue in detail by examining the merger histories of the glob and halo populations.

For objects resolved by 320 or more particles, we can examine the history of mergers involving objects $\geq 10 \%$ of the final object's mass. In practice, the merger history is defined by constructing a "family tree" which recursively traces back in time groups which contain particles that end up in the final object. The procedure is as follows. Consider group $i$ defined at output $n$ (set to the last output frame) consisting of a set of particles $\{p\}$. Find the set of groups $\left\{i^{\prime}\right\}$ at output $n-1$ such that each group has memberships $\left\{p^{\prime}\right\}$ for which the intersection $\left\{p \cap p^{\prime}\right\}$ contains a critical fraction $f_{p}$ of $\left\{p^{\prime}\right\}$. The groups $\left\{i^{\prime}\right\}$ are the progenitors of group $i$. Repeat the process at output $n-2$ to find the set of progenitors for each group in $\left\{i^{\prime}\right\}$. Continue until either no progenitors are found or the earliest output frame is reached. The collection of groups identified at all outputs contains a complete record of merger history of group $i$.

We performed this analysis for the top 35 globs and halos using a critical fraction $f_{p}=0.5$. Results are not terribly sensitive to the choice of $f_{p}$ as long as one avoids the extremes $f_{p}=1$ or 0 . Figure 13 shows the growth histories of the globs with 320 or more particles at the end of the simulation. Isolated systems (those with no other globs within $R_{500}$ of the center of its parent halo) are labeled by brackets in the figure. Globs in the two largest dark matter halos are labeled with asterisks. All other systems shown in the figure are members of smaller groups.

It is clear that the most massive objects have dynamical histories involving a considerable amount of merging. The first and second largest globs lie at the centers of the largest two dark matter halos. The largest object has the most complex formation history, with multiple mergers resulting in a final baryonic mass of $6 \times 10^{11} M_{\odot}$. With such a complex dynamical history, the morphology of the largest object's stellar component would undoubtedly be spheroidal and the final object classified as a giant elliptical or cD galaxy.

Not all merging occurs in group environments, however. Globs 27 and 29, for example, are systems which, although isolated at $z=1$, have experienced significant merger events in the past $10^{9} \mathrm{yr}$. Similarly, not all globs in the two largest groups have undergone extensive merging. Globs 15, 16, and 18 , for example, have had rather clean dynamical histories.

For comparison, we show in Figure 14 the growth histories for the 35 most massive dark matter halos. Halos which contain only one glob out of the top 35 from Figure 14 are labeled by the glob's identification number. The parent halos of globs 27 and 29 have experienced mergers, as expected. However, the times of the halo mergers precede the times when the globs merge by $\gtrsim 10^{9} \mathrm{yr}$. This is somewhat longer, but still of order the infall timescale from a distance of $\sim 100 \mathrm{kpc}$ into the center of the parent halo. Detailed examination of the merger of glob 29 shows a triplet of objects which orbit in a common halo for roughly one crossing time before merging. The behavior is similar to the small group simulations of Barnes (1989).

Comparison of Figures 13 and 14 indicates that the incidence of significant mergers is reduced for the globs relative to the halos. This is quantified in Figure 15, which plots histograms of $\Delta t_{\text {merg }}$, the time elapsed since the last merger involving progenitors whose mass ratio $M_{2} / M_{1}$ exceeds a threshold value $t_{m}$. Histograms for $t_{m}=0.3$ and $t_{m}=0.1$ are shown. Objects incurring no merger satisfying the imposed condition are plotted in the rightmost bin, which is the age of the simulation at $z=1$. For the halos, 30 out of $35(86 \%)$ have experienced a merger with $t_{m}=0.3$, while $33 / 35(94 \%)$ qualify at $t_{m}=0.1$. In contrast, only nine out of the top 35 globs $(26 \%)$ have had a merger at threshold $t_{m}=0.3$ while $19 / 35(54 \%)$ qualify at $t_{m}=0.1$.

One reason for the difference in merging incidence is the reduced cross section of globs relative to halos. The effectiveness of the reduced cross section is illustrated by the fact that 14 of the top 35 dark matter halos contain more than one glob (Fig. 11). In this respect, the effects of treating the galaxies as purely gaseous should be considered. Viscous effects may reduce the cross section of individual objects as well as damp orbital motions - both discouraging and promoting mergers of globs. In addition, one must be cautious about the role played by numerical resolution. Accretion of objects containing only a few tens of particles may be underestimated if the degree of dissipation internal to these small systems is underestimated. However, the large discrepancy between globs and halos for mergers with the larger mass ratio $M_{2} / M_{1}>0.3$ is based on objects resolved by at least 96 particles.

Reduced merger cross sections for galaxies would mitigate arguments against flat, hierarchical cosmologies based on the frailty of thin, galactic disks. Tóth \& Ostriker (1991) have calculated that no more than $4 \%$ of the Milky Way's mass interior to the solar radius could have been accreted via mergers within the last 5 billion yr or else the disk's scale height and Toomre $Q$-parameter would exeeed observed values. They point out that $\Omega=1$ bottom-up clustering models generically 


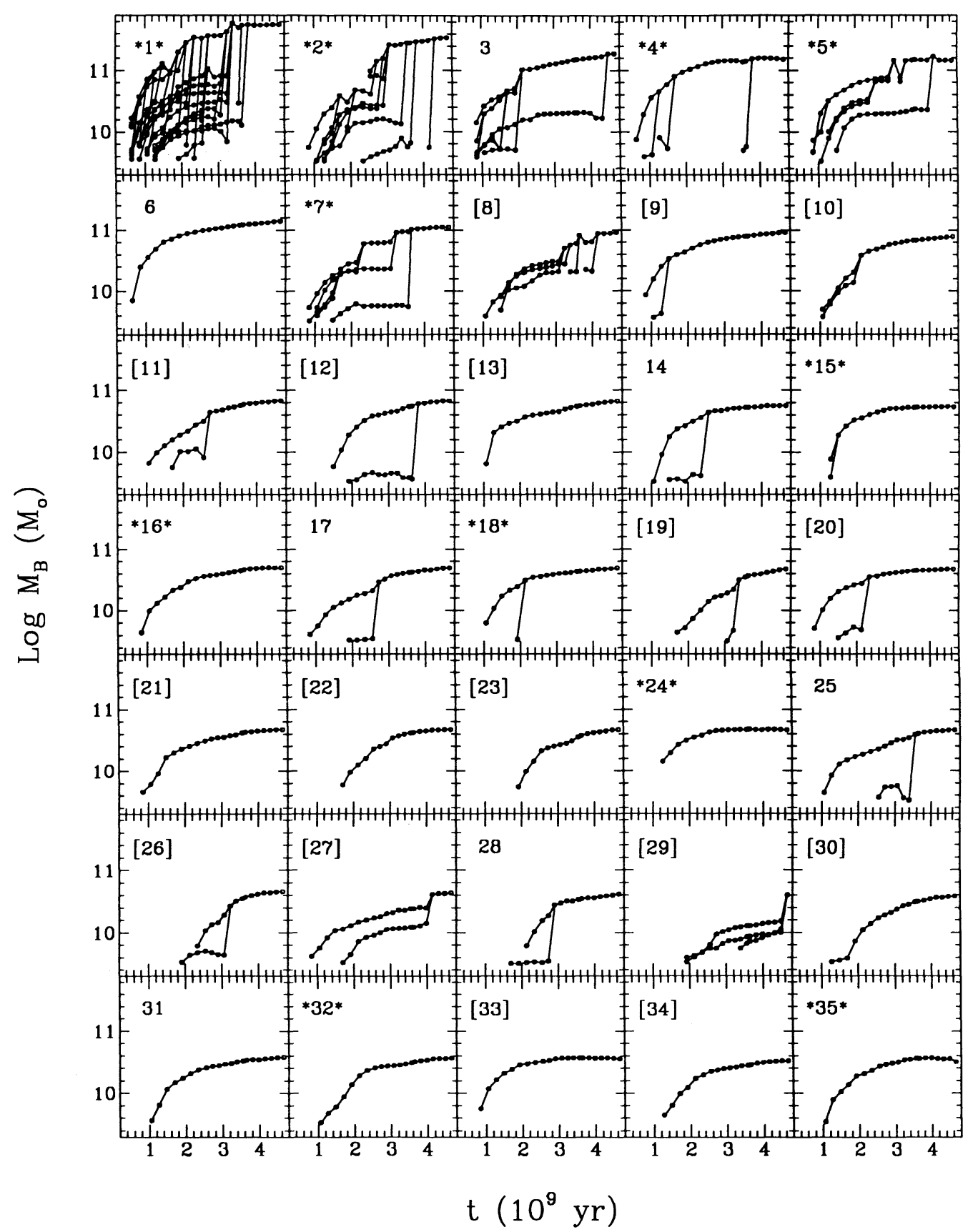

Fig. 13.-Merger histories of the 35 most massive globs found at $z=1$. Numbers indicate mass ranking. Isolated globs are highlighted by [nn] and globs in the two largest dark matter halos are highlighted by *n*.

entail higher merger rates than low $\Omega$ cosmologies and argue that flat models are ruled out by the ubiquitous presence of flat, cold disks in the universe. Their theoretical expectations, based on the model of Carlberg (1990), were that roughly $50 \%$ and $25 \%$ of all galaxies should have suffered mergers involving progenitors with mass ratios $M_{2} / M_{1}>0.1$ and 0.3 , respectively, within the last $\frac{1}{3}$ of a Hubble time. Examining the data in Figure 15, we find that in the last $\frac{1}{3}$ of the age of the simulation, the merger rates for halos were $45 \%$ and $23 \%$, very close to the model expectations. However, the merger rates for globs were suppressed by about a factor 2 , being $23 \%$ and $11 \%$ for the above mass ratios. Although a factor of 2 reduction in merger rate by itself may not be enough to rescue $\Omega=1$ hierarchical models from the disk frailty argument, it does call into question the degree to which we can conclude $\Omega<1$ from merger models.

The upper envelope of the mass histories in Figure 13 can be used to define an average mass accretion rate. Using a simple two-point difference, we define an average rate of accretion from redshift $z$ to the end of the simulation

$$
\left\langle\dot{M}_{B}(z)\right\rangle=\frac{M_{B}(1)-\max \left\{M_{B}(z)\right\}}{t(1)-t(z)},
$$

where $\max \left\{M_{B}(z)\right\}$ is the mass of the largest progenitor at redshift $z$. We take $z=1.5$, for which the time interval in the denominator is $1.3 \mathrm{Gyr}$. Figure 16 shows values of $\dot{M}_{B}$ plotted against the local galaxy density $\Delta_{g}$ determined at $z=1$. Those 


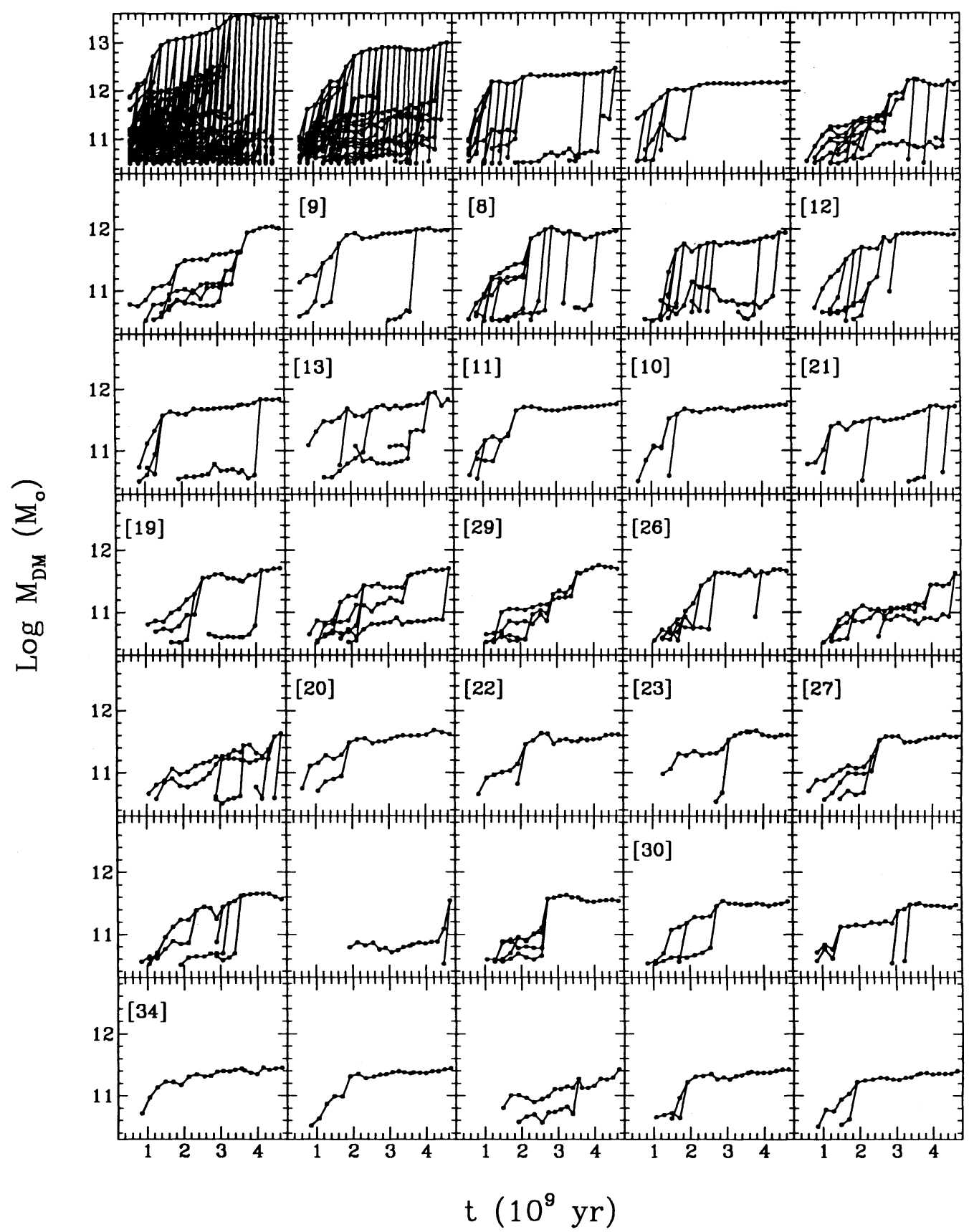

Fig. 14.-Merger histories for the 35 most massive halos found at $z=1$. Note the scale on the top row differs from the others. A halo labeled by an [nn] is the halo containing isolated glob $n n$ shown in Fig. 13.

globs which have experienced a merger above the $t_{m}=0.3$ threshold are plotted as open circles.

Globs which have not experienced a merger grow through accretion at an average rate $\sim 10 M_{\odot} \mathrm{yr}^{-1}$. The accretion rate among this population is reduced in the high-density environment of the large cluster. This reduction is due to ram pressure stripping of the reservoir of material surrounding an individual galaxy once it enters the cluster environment. This evolutionary history follows that proposed by Larson et al. (1980) for the origin of S0 galaxies.

There is weak evidence that major mergers are more likely in high-density environments. All nine globs with density $\Delta_{g}>$ $10^{2}$ are embedded in the two largest dark matter halos. Four out of these nine, or $44 \%$, have had a merger above the $t_{m}=$ 0.3 threshold. Of the globs not in the two largest groups (the "field"), only $21 \%$ have experienced a merger above this threshold. If we assume the major mergers produce predominantly early-type $(E+S 0)$ galaxies, the results are consistent with an enhanced fraction of early-type galaxies in denser environments (Dressler 1980; Postman \& Geller 1984).

\subsection{Morphology, Disk Components, and the Tully-Fisher Relation}

Visual inspection using a sophisticated, three-dimensional graphics package revealed a large number of disk components among the glob population. Figure 17 shows a good example, 


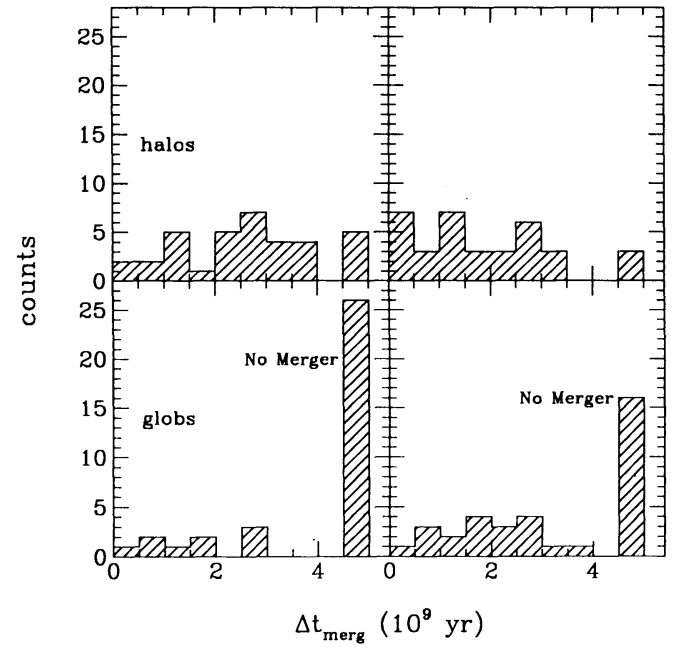

Fig. 15.-Histograms of the time since last merger event $\Delta t_{\text {merg }}$ for the 35 most massive globs (bottom) and halos (top) identified at $z=1$. The left panels are based on mergers between objects with mass ratios $M_{2} / M_{1}>0.3$, while the right panels are based on a $M_{2} / M_{1}>0.1$. The rightmost bin in each histogram corresponds to no merger over the course of the simulation.

the 12 th largest glob at $z=1$. Figure $17 a$ shows the position of the galaxy in the simulated volume. It is located approximately $730 \mathrm{kpc}$ from the center of the main cluster, infalling with a velocity of $850 \mathrm{~km} \mathrm{~s}^{-1}$. Figure $17 \mathrm{~b}$ shows that there is an extended disk component in the cold baryons with a wellordered velocity field extending to $\sim 30 \mathrm{kpc}$ from the center of the galaxy. The disk is slightly warped. The warp most likely arises from differential torques applied during its complicated evolutionary history, similar to that seen in the simulation of Katz (1992). The dark halo, shown out to a $100 \mathrm{kpc}$ distance from the center, is smooth and slightly oblate in the inner parts.

We investigated the shapes of both the globs and their dark matter halos for isolated systems with more than 320 particles. With such a relatively small number of particles, a detailed look at shapes as a function of radius is not possible. Instead, we perform a straightforward moment of inertial analysis. For the 17 globs, we calculate the inertia tensor of the cold $\left(T<10^{5} \mathrm{~K}\right)$ material within a $30 \mathrm{kpc}$ radius. We diagonalize the inertia tensor and rank the moments $I_{1} \geq I_{2} \geq I_{3}$. The

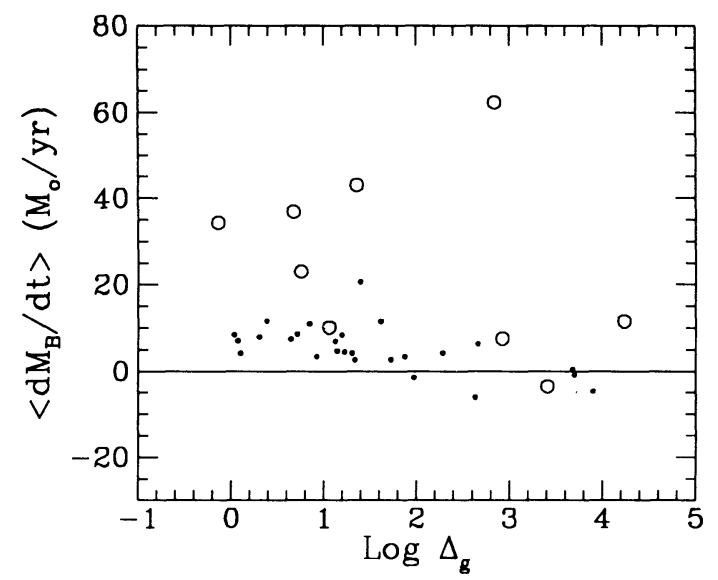

FIG. 16.-The mean mass accretion mass rate for globs over the $1.3 \mathrm{Gyr}$ from $z=1.5$ to $z=1$ against the final local galaxy density. Circles show globs which have experienced a merger with $M_{2} / M_{1}>0.3$. ratios of the moments $I_{3} / I_{2}$ versus $I_{2} / I_{1}$ are plotted in Figure 18. Prolate objects would lie on the right edge of the plot $\left(I_{2} / I_{1}=1\right)$ while oblate objects would lie along the top $\left(I_{3} / I_{2}=1\right)$. A sphere would lie in the upper right corner. Since a thin disk is an extreme case of an oblate spheriod, it would occupy the upper left corner of Figure 18.

Almost all of the globs occupy the disk corner of the plot, with 15 of the 17 clustered strongly about $I_{2} / I_{1}=0.65$ and $I_{3} / I_{2}=0.85$. The two outliers, globs 13 and 29 , are both dynamically peculiar. Glob 13 has a small satellite just below the 32 particle cutoff which lies about $30 \mathrm{kpc}$ from its center. Glob 29 is the product of a recent triple merger (see Fig. 13). Visual inspection verifies that the well-resolved, isolated globs exhibit distinct disks.

Using high-resolution collisionless simulations, Dubinski \& Carlberg (1991) found the shapes of dark matter halos in the CDM model to be significantly flatter than the observed shapes of elliptical galaxies. A loophole in this discrepancy (besides the fact that the stellar component may differ from the dark halo in shape) is the idea that dissipation of a baryonic component into the center of the halo could modify its structure. We investigate this question by comparing the shapes of halos in the gas dynamical simulation with shapes determined from the dark matter-only run employing the same initial conditions.

For the halos, we reran the group-finding algorithm using $\eta=0.06$ in order to examine the shape of the inner parts of halos above a density contrast of $\sim 5000$. The shapes of the halos with more than 100 particles associated with the isolated globs in the two-fluid run are shown as open circles in Figure 18. Their counterparts in the single-fluid run are plotted as filled circles. Lines join the common halo pairs. Halos in the run with baryons are generally more spherical than the corresponding halos in the run with no baryons. The centroid of the distributions shifts from $(0.84,0.53)$ for DM only to $(0.88,0.65)$ for $\mathrm{DM}+\mathrm{B}$. The inferences one can draw from these experiments are limited by the small number of objects, modest mass resolution, and lack of a stellar component. What we can conclude is that the influence of the baryonic component cannot be ignored in any analysis of the inner shapes of dark matter halos. It is obviously crucial to understanding the morphological characteristics of the stellar component as well.

The moment analysis indicates that the dissipated baryons are much more "disklike" than their parent halos. The question naturally arises whether the disk component is a numerical transient or a stable, centrifugally supported system. To address this question, we examine components of the velocity field in a cylindrical coordinate system with $z$-axis aligned with the largest eigenvector determined from the moment analysis. The velocity fields of the cold gas in the 17 isolated, massive globs are shown in Figure 19. The points indicate the mean tangential component $v_{t}$ with error bars showing the dispersion about the mean in radial bins containing 20 particles each. The dotted and dashed lines give the mean radial and vertical components, respectively. The solid line gives the equilibrium value $v_{\text {circ }}$ of the circular rotation speed based on the enclosed mass profile for the softened Plummer potential

$$
v_{\text {circ }}(r)=\sqrt{\frac{G M_{\mathrm{tot}}(<r) r^{2}}{\left(r^{2}+\epsilon^{2}\right)^{3 / 2}}} .
$$

Profiles are traced to the maximum extent of the cold $(T<$ $\left.10^{5} \mathrm{~K}\right)$ material within a maximum of $30 \mathrm{kpc}$. 


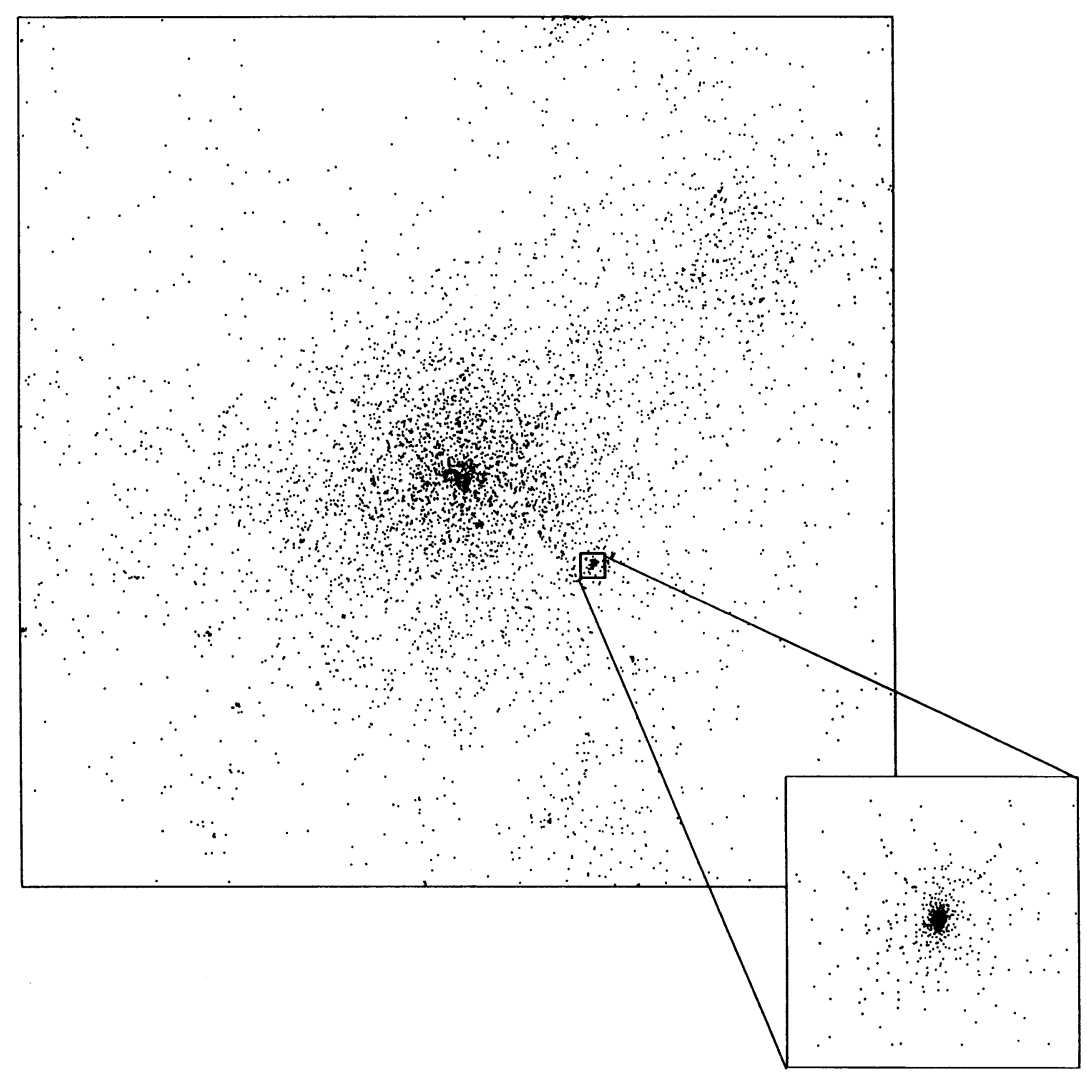

FIG. $17 a$
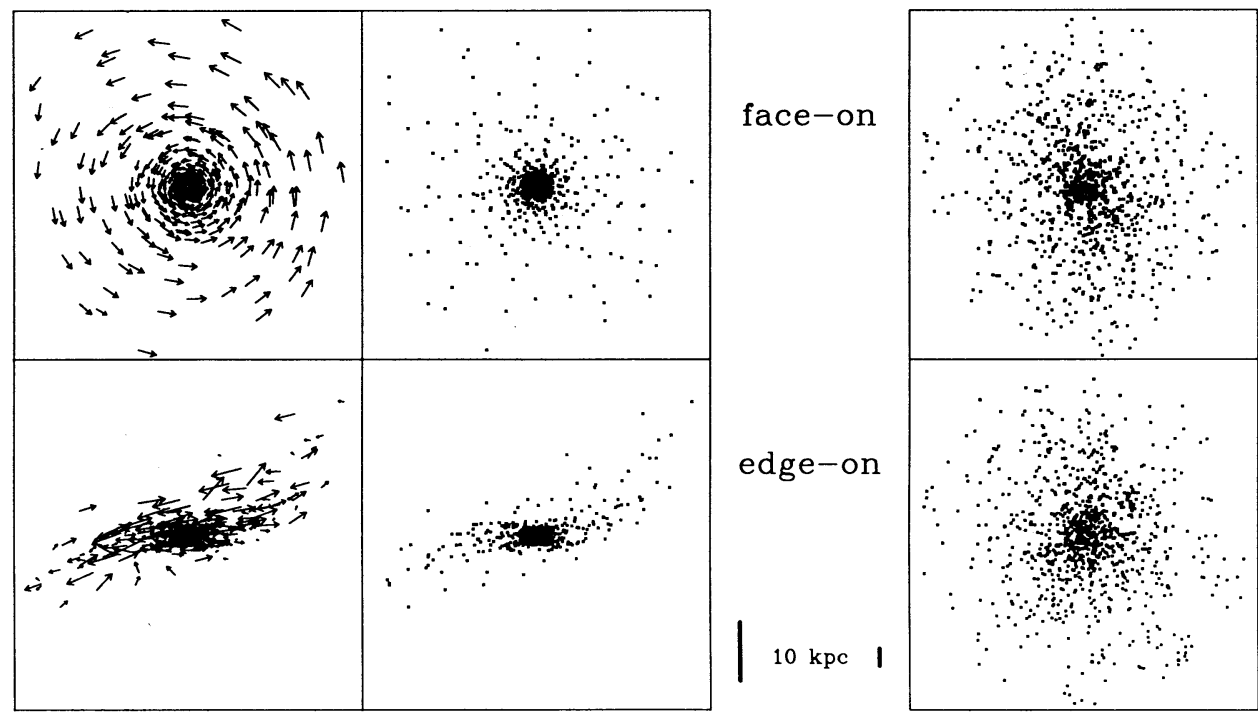

FIG. $17 b$

FIG. 17. $-(a)$ Environment of the 12th largest galaxy found at $z=1$. (b) Close-up view in the principal component frame. The upper and lower rows show face-on and edge-on views, respectively. The panels on the left show baryons with $T<3 \times 10^{4} \mathrm{~K}$ within a cubic region $60 \mathrm{kpc}$ on a side centered on the glob. The velocity field of the baryons shows orderly circulation of the disk at a velocity of $200 \mathrm{~km} \mathrm{~s}^{-1}$ with little motion out of the plane. The right-hand column shows the dark matter particles within a radius of $100 \mathrm{kpc}$ of the center. Vertical bars indicate a spatial scale of $10 \mathrm{kpc}$, roughly the resolution limit of the simulation. 


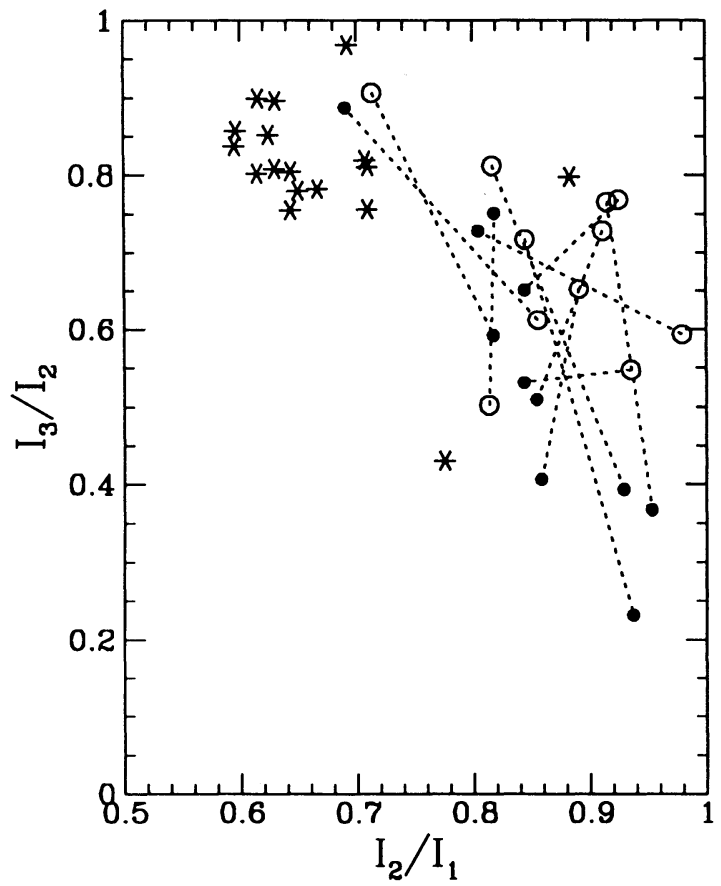

FIG. 18.-Shapes of isolated objects within the top 35 most massive identified at $z=1$. The axes are the ratios of the eigenvalues of the moment of inertia tensor $I_{1}>I_{2}>I_{3}$. Symbols represent: globs (asterisks); halos in the two-fluid run (circles); and halos in a collisionless-only run (filled circles). Only halos common to the top 35 in both runs are shown, joined by dotted lines. Globs are more disklike than halos, and halos in the two-component run are rounder than their counterparts in the collisionless run.

All of the disks are very nearly rotationally supported. An obvious exception is glob 29, the recent triple merger. Also, the satellite companion of glob 13 affects the rotation curve near $30 \mathrm{kpc}$. The outer parts of glob 19 are affected by recent accretion of an object below the 32 particle cutoff. The merger is evident in its halo mass history shown in Figure 14 because of the lower density threshold used for halo material. Also shown in the lower right corner is the velocity field of glob 28 . This object, with a baryonic mass $M_{B}=4 \times 10^{10} M_{\odot}$, is the main member of a triplet composed of itself and two small companions, each roughly $\frac{1}{10}$ this mass. It is an example of an extended, rotationally supported disk in a multiple system similar in scale to the Milky Way and Magellanic Clouds.

Widening our analysis to globs with 100 particles or more in both isolated and multiple systems, we show in Figure 20 a plot of

$$
f_{\text {rot }}=\frac{v_{t}}{v_{\text {circ }}}
$$

evaluated at the maximum value of $v_{t}$ in the rotation curve. The quanitity $f_{\text {rot }}$ is an estimate of the degree of rotational support of the cold baryons. Again, isolated objects are plotted using filled circles and nonisolated globs with open circles. The dashed lines marking the interval $0.1<f_{\text {rot }}<0.25$ denote the regime in which dark halos fall under similar analysis. Like elliptical galaxies, halos are not supported by rotation.

For isolated globs, there is a trend of decreasing $f_{\text {rot }}$ toward smaller masses. This is likely to be due to lack of resolution of a disk component in low-mass objects. For all systems, the fraction of mass in an extended disk component is small, $\$ 30 \%$.
This result was apparent in the earlier work of Katz \& Gunn (1991) and Navarro \& Benz (1991). Both of these numerical studies, which employed a similar SPH algorithm to perform the gas dynamics, grew galaxies in which a large fraction of the baryonic mass ended up in an unresolved, central component. This problem was partly allieviated in further simulations by Katz (1992) which incorporated star formation. As a result of the peaked central mass concentrations, the extended disks of the smaller globs are resolved by only a few tens of particles. Both the dynamical evolution and the subsequent moment analysis will be noisier for smaller systems. This produces the trend toward lower $f_{\text {rot }}$ for isolated globs in Figure 20.

The nonisolated globs show large scatter in $f_{\text {rot }}$, a direct result of mergers and close encounters with neighboring systems. In general, extended disk components are found in the subset of systems which lie in the same region of the plot as the isolated globs. Disks appear to be abundant when they can be resolved.

Do these disks obey something like a Tully-Fisher relation? In Figure 21, we plot the baryonic mass against measured rotation velocity for a subset of the isolated globs shown in Figure 20. To select the most likely candidates for disk galaxies, we have imposed a cutoff $f_{\text {rot }}>0.7$, which translates into more than half the kinetic energy of the extended component being in ordered rotation. This results in 28 objects spanning a decade in mass from $10^{10}$ to $10^{11} M_{\odot}$. The open circles in Figure 21 use the measured maximum of the rotation curve. A least-squares fit to a power law yields $M_{B}=2.7 \times 10^{10}\left(v_{\text {rot }} / 100\right.$ $\left.\mathrm{km} \mathrm{s}^{-1}\right)^{1.7}$. The scatter about the best-fit relation is small; the rms deviation $\Delta$ in $\log M_{B}$ at a given velocity is 0.10 , or 0.25 mag if $L \propto M_{B}$.

To compensate for the poorer numerical resolution in smaller disks, we apply a correction enforcing rotational support, letting $v_{\text {rot }}^{c}=v_{\text {rot }} / f_{\text {rot }}$. The asterisks in Figure 21 use the corrected rotation velocity $v_{\text {rot }}^{c}$. The dotted line in the figure shows the best-fit line $M_{B}=1.7 \times 10^{10}\left(v_{\text {rot }}^{c} / 100 \mathrm{~km} \mathrm{~s}^{-1}\right)^{2.5}$. The scatter in this relation is tighter than for the raw velocities, with $\left\langle\Delta^{2}\right\rangle^{1 / 2}=0.05$, or $0.13 \mathrm{mag}$.

Because centrifugal equilibrium is enforced, the $M_{B} \propto v^{2.5}$ relation shows the correspondence of the baryonic mass to the mass profiles found in our simulation. For the fiducial mass profile of $M(r) \propto r$, measuring the velocity at a fixed radius yields $M \propto v^{2}$. If one measured the velocity at a fixed density threshold instead $\left(r \propto M^{-1 / 3}\right)$, a relation of $M \propto v^{3}$ would result. Our slope of 2.5 using a measured mass profile and measuring the velocity at the peak of the curve is clearly in the expected regime. However, the mass profile is dominated at large radii by the dark matter while the luminosity of a galaxy stems from its baryonic content. The differences from the above relations indicate the systematic variation of baryonic mass to total mass at $30 \mathrm{kpc}$.

What is most interesting is the small degree of scatter in the experimental $M_{\text {glob }}-v_{\text {rot }}$ relation. It is not obvious that such a small scatter should arise naturally. One might have thought that the variety of dynamical histories expected in Gaussian random models could lead to larger scatter (Kaiser 1988). If the scatter had been larger than the $\sim 0.3 \mathrm{mag}$ observed, one would have had to rely on the effects of star formation or other processes to eliminate some of the scatter, a daunting prospect indeed. Instead, we have some breathing room to add extra scatter when producing an $L-v$ relation.

The slope of 2.5 between mass and velocity could be reconciled with an $L \propto v^{\beta}$ relation if one assumes the star formation 


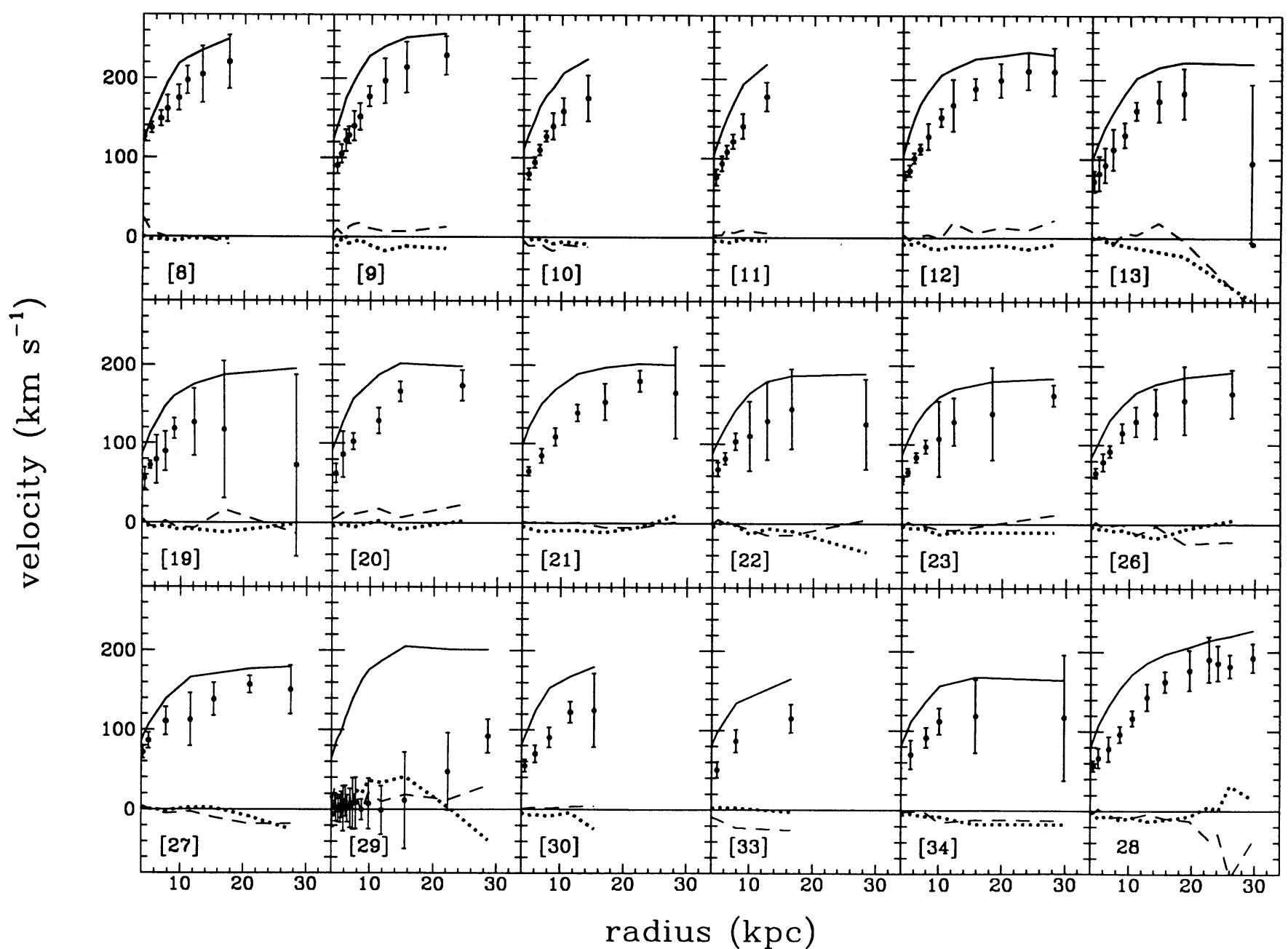

Fig. 19.- Measured rotation curves for isolated, massive galaxies. Data points and error bars show the mean tangential velocity $v_{t}$ and the dispersion in $v_{t}$ binned in radii containing 20 particles in each bin. Dotted and dashed lines show the mean radial and vertical velocity components, respectively. Solid lines are the equilibrium estimate of $v_{\text {rot }}$, eq. (14). Glob 28 in the lower right is a member of a triplet.

efficiency varies in such a way as to produce a baryonic massto-light ratio scaling as

$$
\frac{M_{B}}{L} \propto L^{-1+2.5 / \beta}
$$

For spirals, it has been argued that the value of $\beta$ depends both on galaxy type and on the chosen bandpass (Rubin et al. 1982; Djorgovski, De Carvalho, \& Han 1989). Using the fiducial value $\beta=4$, we would require

$$
\frac{M_{B}}{L} \propto L^{-0.38}
$$

For extreme values of $\beta=3$ and 5 , the required slopes in $M_{B} / L$ against $L$ would be -0.17 and -0.50 , respectively. In all cases, the baryonic mass-to-light ratio increases for smaller galaxies. Indirect observational support for such a trend comes from the fact that the ratio of dynamical mass to light increases toward smaller galaxies (Persic \& Salucci 1988). Dwarf galaxies such as DDO 154 (Carignan \& Freeman 1988) may be the extreme end of this trend. On the other hand, existence of the rare massive, gas-dominated disk systems such as Malin 1 (Impey,
Bothun, \& Malin 1988) must call into question any scheme advocating efficient star formation in all massive galaxies.

It is interesting to examine the impact of equation (17) on the faint end slope of the luminosity function derived from the mass function. If $M_{B} \propto L^{\zeta}$, then the faint end slope transforms

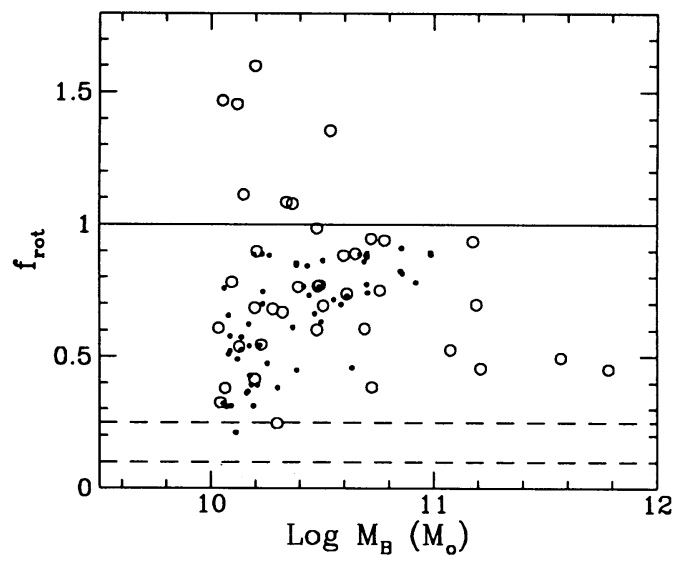

FIG. 20.-Rotational support measure $f_{\text {rot }}$ against mass for globs resolved by 100 particles or more. Circles show globs in groups and dots are isolated. 


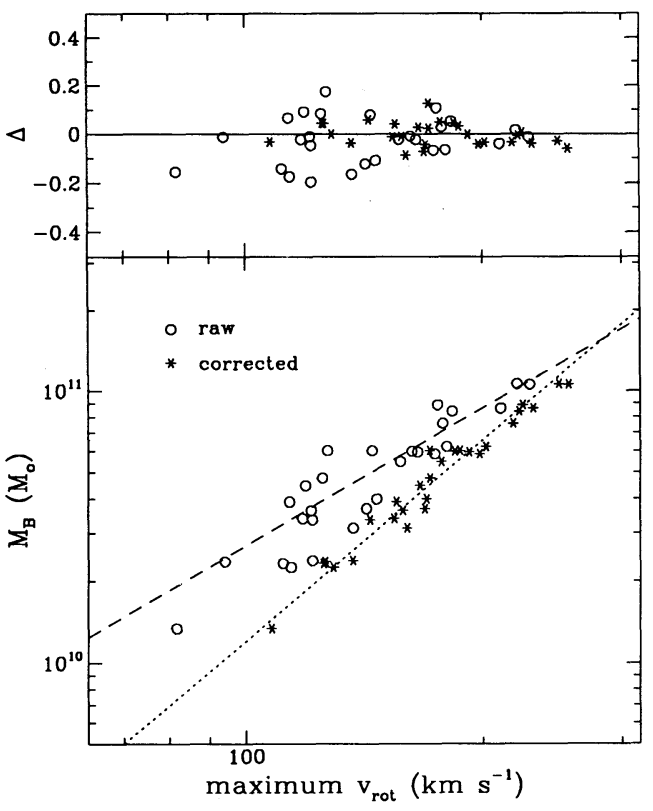

Fig. 21.-Glob mass plotted against the maximum rotation velocity (equivalent of the Tully-Fisher relation) for isolated systems from Fig. 20 with $f_{\text {rot }}>0.7$. Open circles use the raw $v_{t}$ as a direct indicator of $v_{\text {rot }}$. Asterisks employ a corrected estimate $v_{\text {rot }}=v_{t} / f_{\text {rot }}$. The best-fit lines of slopes 1.7 (dashed) and 2.5 (dotted) are shown. The upper panel shows the residual in $\log M_{B}$ about the best-fit relations. The rms residuals in $\Delta$ are 0.10 and 0.05 for the raw and corrected estimates, respectively.

as $1+\alpha_{L}=\zeta\left(1+\alpha_{g}\right)$, so the value of $\alpha_{g}=-1.39 \pm 0.12$ measured in the simulation implies

$$
\alpha_{L}=-1.24 \pm 0.07,
$$

which is still steeper than the recent APM estimate but agrees well with the earlier determinations of Sandage et al. (1979) and Kirshner et al. (1983). The above analysis is certainly speculative, but it is worth noting that the star formation efficiency of equation (17) may solve two independent problems simultaneously.

\subsection{Correlation Properties and Biasing}

Understanding the links between the observed galaxy distribution and the underlying distribution of dark matter is another motivating factor behind gas dynamic simulations. The most common assumption presently employed is that galaxies are spatially biased with respect to the dark matter. That is, the spatial two-point correlation function for the galaxies $\xi_{g g}(r)$ is an enhanced version of the corresponding function for the dark matter $\xi_{\rho \rho}$ (see Davis et al. 1985). The degree of bias is characterized by a factor $b$ defined as the ratio of the rms amplitude of density fluctuations, $\sigma=\left\langle(\delta \rho / \rho)^{2}\right\rangle^{1 / 2}$, for galaxies and dark matter on a scale of $8 h^{-1} \mathrm{Mpc}$

$$
b=\left.\frac{\sigma_{g g}}{\sigma_{\rho \rho}}\right|_{8 h^{-1} \mathrm{Mpc}} .
$$

With $\sigma_{g g}$ determined from galaxy surveys (Davis \& Peebles 1983), the bias parameter sets the amplitude of fluctuations in the dark matter component.

We show in Figure 22 the correlation functions for globs and dark matter at two epochs, $z=3.0$ and $z=1.0$. Two mass thresholds are used for the galaxies, 32 and 256 particles, corresponding to $M_{B}>0.35$ and $2.8 \times 10^{10} M_{\odot}$, respectively. Also shown is the observed correlation function scaled to redshift $z$ assuming stable clustering

$$
\xi_{\text {obs }}(r)=\left(r / r_{0}\right)^{-\gamma}(1+z)^{-3+\gamma},
$$

where $r$ is a comoving separation, $r_{0}=5 h^{-1} \mathrm{Mpc}$, and $\gamma=1.8$ (Peebles 1980). We show this curve as a guide. Because of the central cluster, the statistics in the small volume simulated are not a fair sample of the overall level of clustering expected in a CDM universe. To address the issue of biasing, we are concerned only with the relative clustering properties of the globs and dark matter.

For either mass threshold, the globs at $z=3$ are more clustered than the dark matter. A mass dependence in $\xi_{g g}$ is clearly apparent; clustering of the high-mass sample is enhanced by a factor $\sim 4$ over the low-mass sample. By $z=1$, the dark matter appears to have "caught up" with the galaxies and, in fact, the low-mass globs are somewhat less clustered than the dark matter ("negatively" biased) on scales $\gtrsim 300 \mathrm{kpc}$. The same behavior was reported by Couchman \& Carlberg (1992), who argued that the suppression of clustering in the galaxy population was due to merging of galaxies in rich clusters. However, since their method cannot follow galaxy tracers in the cores of

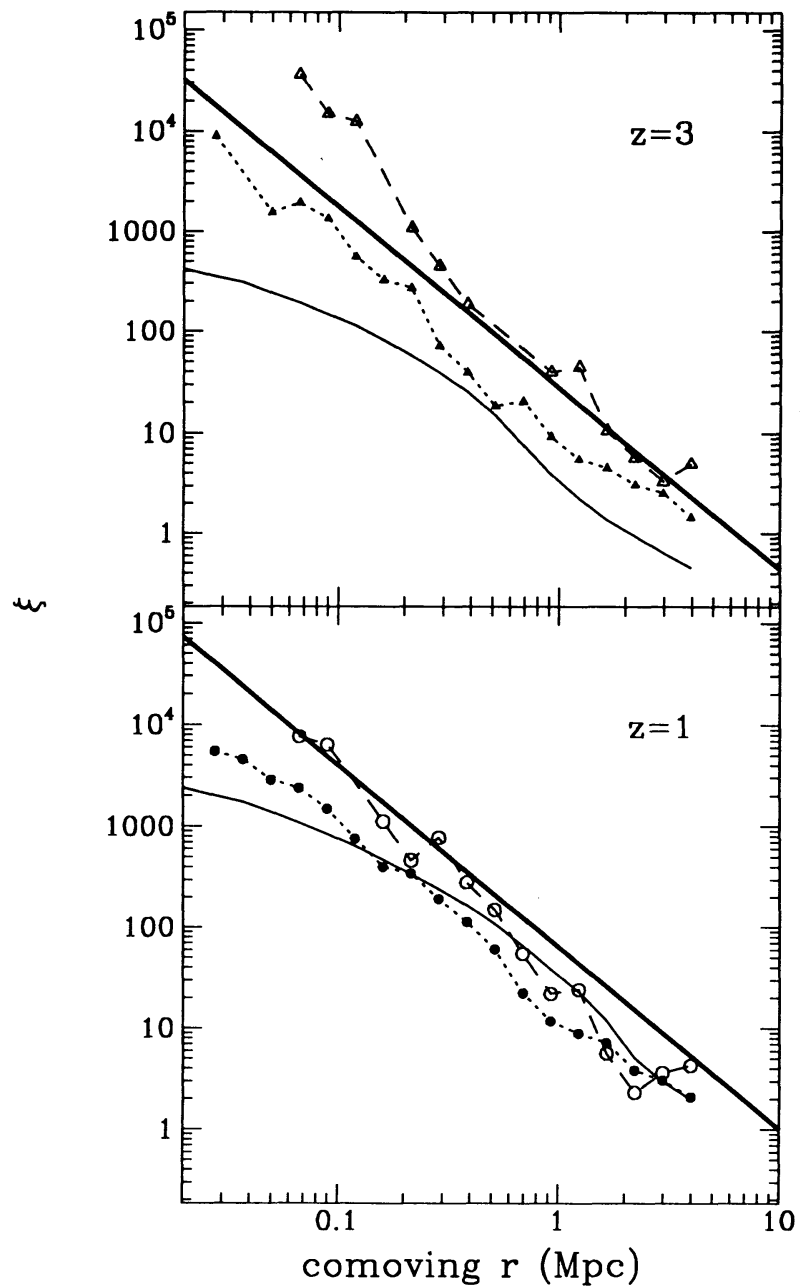

Fig. 22.-Two-point correlation function for the dark matter (solid line), all globs with $M_{B}>3.5 \times 10^{9} M_{\odot}$ (filled symbols), and massive globs with $M_{B}>$ $2.8 \times 10^{10} M_{\odot}$ (open symbols) at $z=3$ and $z=1$. The heavy line in both panels is observed galaxy correlation function scaled assuming stable clustering. 
clusters (Summers et al. 1993) and they exclude objects above a mass of $4 \times 10^{12} M_{\odot}$, such a conclusion is premature. None$\therefore$ theless, we find below that this is the most likely explanation for the behavior in our experiment.

To quantify the degree of biasing as a function of scale, we follow Katz et al. (1992) and use the ratio of the volume integrals of $\xi(r)$

$$
b_{\xi}(r)=\left[\frac{\int_{0}^{r} d x x^{2} \xi_{g g}(x)}{\int_{0}^{r} d x x^{2} \xi_{\rho \rho}(x)}\right]^{1 / 2} .
$$

Figure 23 shows $b_{\xi}(r)$ based on the correlation functions shown in Figure 22. The behavior at $z=3$ on scales $\gtrsim 500 \mathrm{kpc}$ is in quite good agreement with a constant bias. Values of $b_{\xi}=1.7$ and 3.0 are seen for the low- and high-mass samples, respectively. Enhanced clustering of more massive galaxies was also seen in the experiments of Carlberg \& Couchman (1989) and Katz et al. (1992) and likely reflects a connection between initial perturbations height and final mass in the sense that the most massive galaxies arise from higher perturbations in the density field which are inherently more strongly clustered in Gaussian random fields (Bardeen et al. 1986). By $z=1$, the high-mass sample is more clustered than the dark matter below $\sim 1 \mathrm{Mpc}$ but is nearly equally clustered (unbiased) beyond this scale. The low-mass sample is seen to be negatively biased on scales $\gtrsim 300 \mathrm{kpc}$. The minimum value of $b_{\xi} \simeq 0.7$ is reached at $1 \mathrm{Mpc}$.

We note that although the Katz et al. study claims a value of $b_{\xi}=1.5$ at $8 \mathrm{Mpc}$, their Figure 4 indicates that the galaxies and dark matter have nearly equal clustering amplitude over the range $0.1-2 \mathrm{Mpc}$. Because of the lower mass resolution in their experiment, their correlation function should be compared to that of our massive sample. The two studies seem to agree with a modest level of biasing in the quoted spatial range. The study of Cen \& Ostriker (1992b) found a spatial bias of $\sim 1.6-2$ over the range 1-2 $10 \mathrm{Mpc}$. They used a method to locate galaxies which is very different from that employed by the SPH studies.

To investigate the source of the antibias measured at $z=1$, we performed a counts-in-cells analysis for both dark matter

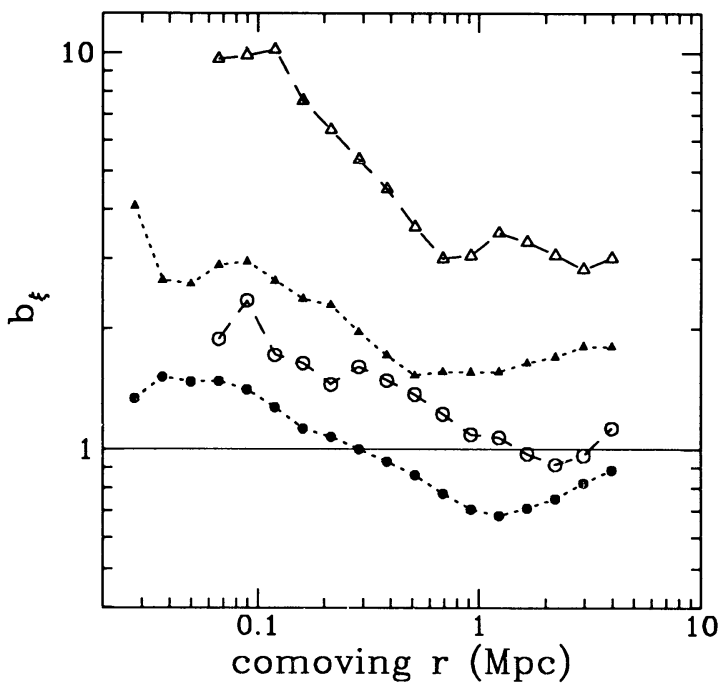

FIG. 23.-Spatial bias parameter $b_{\xi}$, eq. (21), based on the correlation functions of Fig. 24. Symbol types are as in that figure, triangles are data at $z=3$, and circles are $z=1$. Filled symbols are all globs and open symbols show the massive subsample.

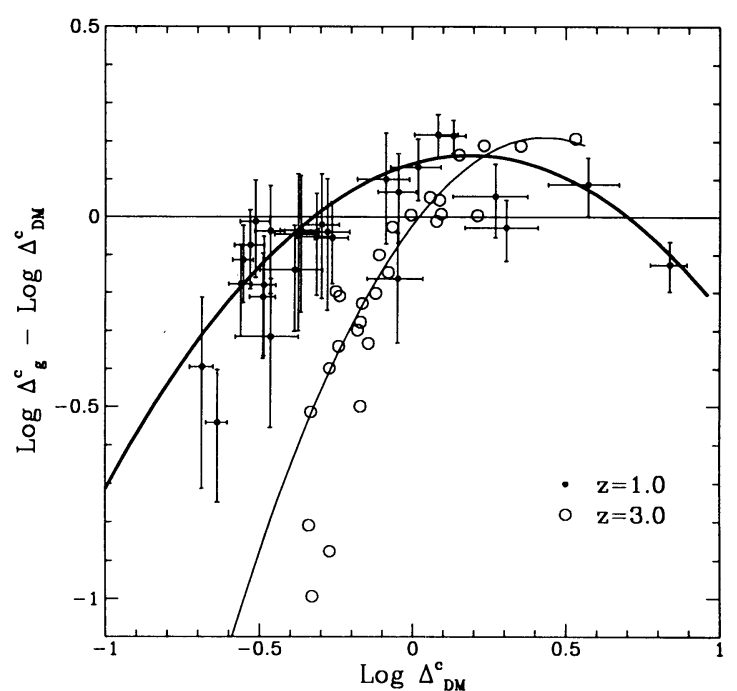

FIG. 24.- Log of the ratio of density contrasts in galaxies and dark matter determined by counts in cells on a comoving scale $L / 3=5.3 \mathrm{Mpc}$. Filled circles are data at $z=1$, open circles are $z=3$ (error bars omitted for clarity). The straight line is $\Delta_{g}=\Delta_{\mathrm{DM}}$ (i.e., no spatial bias) while the bold and thin lines show quadratic fits, eq. (22).

and galaxies in cells of comoving side $L / 3=5.3 \mathrm{Mpc}$. Within each cell, we measure the density contrast $\Delta^{c}=N /\langle N\rangle$, where $\langle N\rangle$ is the expected number of dark matter particles or globs in a cell based on the mean number density. In this way, we can examine directly the relationship between fluctuations in dark matter and glob counts. The results are shown in Figure 24 for $z=3$ and 1 . Error bars were generated by systematically varying the cell center by half a cell width in each direction, leading to a total of eight density estimates per cell. The data were fitted to a quadratic in $\log (\Delta)$

$$
\log \left(\Delta_{g}^{c}\right)=a_{0}+a_{1} \log \left(\Delta_{\mathrm{DM}}^{c}\right)+a_{2}\left[\log \left(\Delta_{\mathrm{DM}}^{c}\right)\right]^{2} .
$$

Parameters from the fit are shown in Table 5.

The coefficient $a_{1}$ is another estimator of the linear bias parameter, since writing $\Delta^{c}=1+\delta^{c}$ and expanding equation (22) to first order in $\delta^{c}$ gives

$$
\delta_{g}^{c}=a_{1} \delta_{\mathrm{DM}}^{c}
$$

if the DC coefficient $a_{0}=0$, as it must be for a linear bias. The data at $z=3.0$ are consistent with this assumption, while the data at $z=1.0$ favor a nonzero value for $a_{0}$, inconsistent with linear biasing. Still, calling this estimator of the spatial bias $b^{c}$, we find from Table 5 values of $b^{c}=2.08 \pm 0.40$ at $z=3$ and $b^{c}=1.23 \pm 0.25$ at $z=1$. The quoted errors are the formal values from the fitting routine multiplied by a conservative factor of $\sqrt{8}$ to account for the fact that the data giving rise to the points of Figure 24 are oversampled by a factor 8 .

As in the correlation function estimate, the spatial bias decreases with time. However, there is no evidence in the count in cells analysis for galaxies being antibiased except for the

TABLE 5

Counts-in-Cells Fitting Parameters

\begin{tabular}{crcc}
\hline \hline$z$ & \multicolumn{1}{c}{$a_{0}$} & $a_{1}$ & $a_{2}$ \\
\hline $3.0 \ldots \ldots \ldots$ & $-0.02 \pm 0.03$ & $2.08 \pm 0.14$ & $-1.26 \pm 0.37$ \\
$1.0 \ldots \ldots \ldots$ & $0.14 \pm 0.04$ & $1.23 \pm 0.09$ & $-0.62 \pm 0.19$ \\
\hline
\end{tabular}


highest density cell containing the large cluster at $z=1$. At $z=3$, before the bulk of the cluster collapsed, the globs are positively biased throughout the volume. We infer that merging of galaxies in the cluster is largely responsible for the antibias signal in the correlation function. Although the glob merger cross sections are certainly smaller than those of their dark matter halos, they are still somewhat larger than those of real galaxies, since our resolution limit is somewhat larger than the characteristic scale of the visible mass in galaxies. The fact that they remain collisional throughout their history also increases their merger rate. We suspect that the antibias signal is unphysical. We are currently investigating this issue using a pair of simulations designed to compare collisional and collisionless treatment of globs.

Along with biasing in the spatial domain, galaxies may be biased relative to dark matter in velocity space. The idea of velocity biasing was hinted at in the original CDM experiments of Davis et al. (1985) and was examined for groups of galaxies by Evrard (1987). The latter study compared the velocity dispersion of galaxy groups with and without dark halos and found that galaxies within groups had velocity dispersions reduced by a typical factor of 0.8 relative to the dark matter. The same value was found in the study of a single cluster by Carlberg \& Dubinski (1991). Studies by Carlberg, Couchman, \& Thomas (1990) and Couchman \& Carlberg (1992) saw a much larger effect. Their estimates of the velocity bias parameter $b_{v}(r)$, defined as the ratio of rms pairwise velocity dispersions of galaxies and dark matter with separation $r$, encompassed the range $0.3-0.5$ on scales $\sim 1 \mathrm{Mpc}$. However, Carlberg (1991) quotes a value of 0.7 as appropriate for galaxies. To make a confusing matter even murkier, the recent Katz et al. (1992) paper claims to see no velocity bias on the same scales addressed by the previous experiments while Cen \& Ostriker (1992b) see an effect of $\sim 0.8$ at $1 \mathrm{Mpc}$.

We now add our results to the discussion. Figure 25 shows the rms pairwise peculiar velocities as a function of separation at $z=3$ and $z=1$ for dark matter, low- and high-mass globs. The glob velocities are clearly lower than the dark matter values on small scales, but become comparable on comoving scales $\gtrsim 3 \mathrm{Mpc}$. At $z=1$, the amplitude of the glob velocity dispersion is fairly constant at $\sim 400 \mathrm{~km} \mathrm{~s}^{-1}$ on scales $\gtrsim 100$ $\mathrm{kpc}$. This is larger than the present observed value of $\sim 300 \mathrm{~km}$ $\mathrm{s}^{-1}$ (Davis \& Peebles 1983; Bean et al. 1983).

The velocity bias, the ratio of glob to dark matter velocity dispersions, is shown in Figure 26. The amplitude of $b_{v}$ depends on spatial scale but appears to be only mildly dependent on redshift and glob mass. A rough power-law fit is given by $b_{v}(r)=0.7(r / 1 \mathrm{Mpc})^{0.2}$. At $0.1 \mathrm{Mpc}$, the effect is fairly significant, $b_{v} \simeq 0.5$. The lack of dependence on epoch and mass seems to downplay the role of dynamical friction in producing a velocity bias, as proposed by Carlberg et al. (1990). One would expect dynamical bias to increase with time (lower $b_{v}$ ) and to be stronger for more massive galaxy tracers if dynamical fricition in a fixed background were the cause. Though Carlberg et al. find both effects, our results are consistent with only a weak effect on these scales.

Another source of reduced velocities is the fact that galaxies are preferentially formed at sites of local potential minima. They are born "cold" with respect to their parent halos, which implies they start out strongly biased toward sites of high binding energy. The relaxation process in hierarchical models is known to be incomplete in the sense that binding energy is not forgotten as each stage of the clustering hierarchy advances

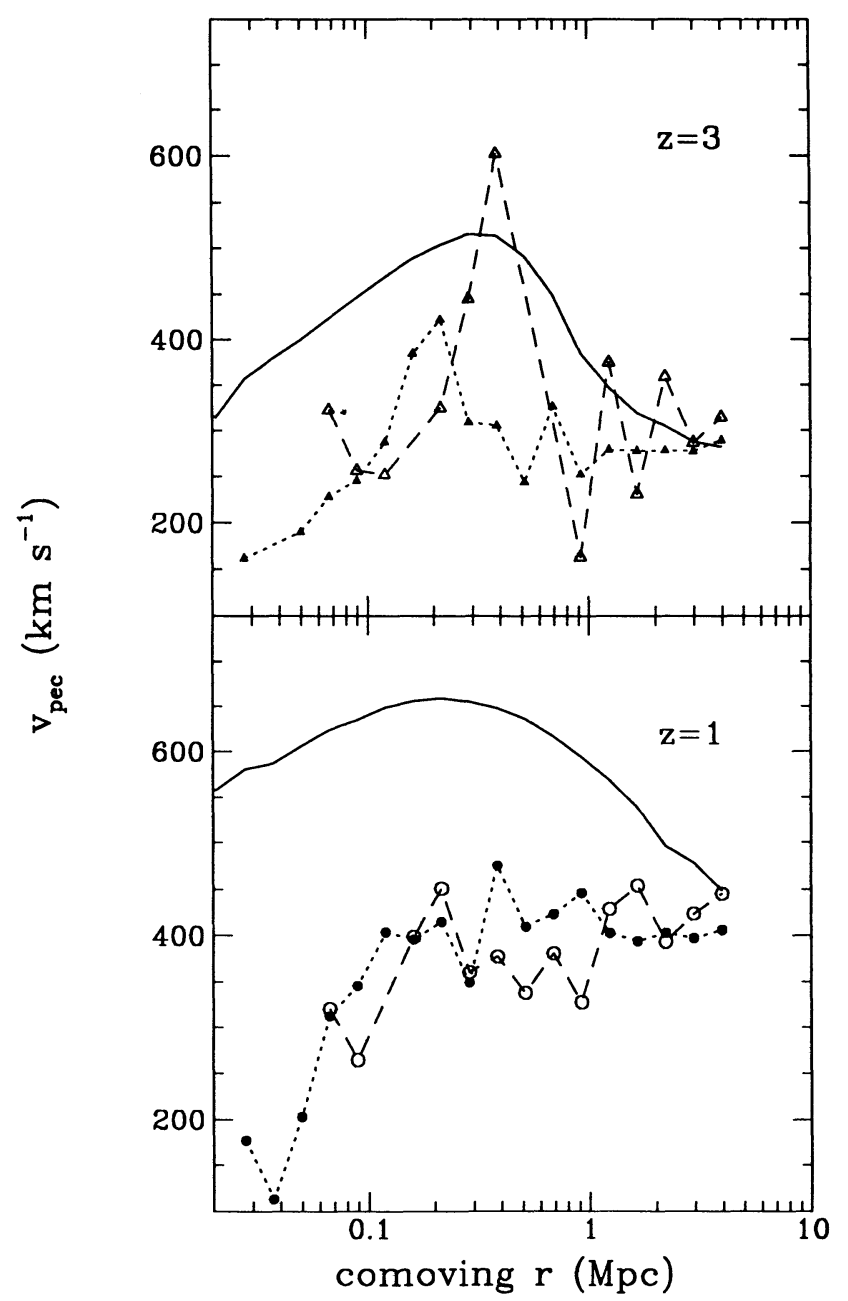

FIG. 25.-Mean one-dimensional pairwise peculiar velocity as a function of pair separation. Symbols are as in Fig. 24.

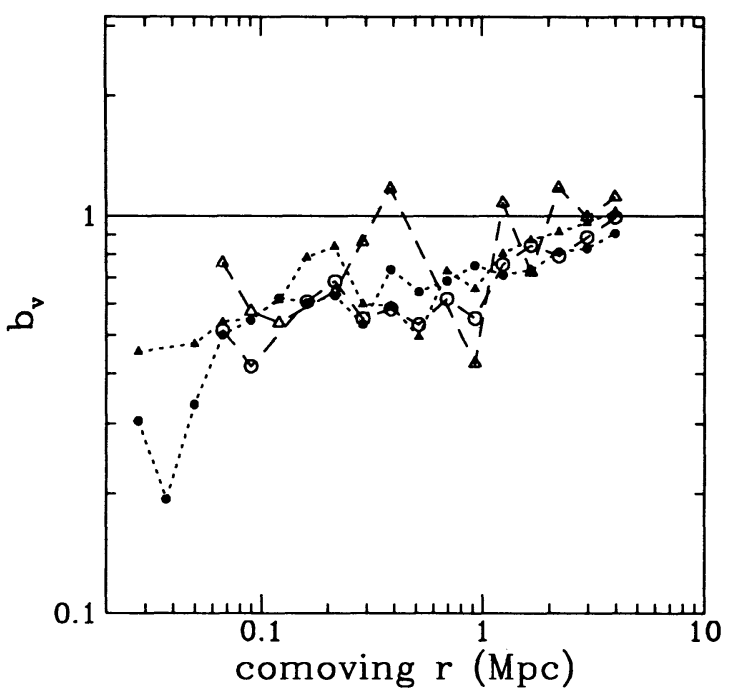

FIG. 26.-Velocity bias parameter $b_{v}$, defined as the ratio of galaxy to dark matter pairwise velocity, as a function of scale. 
(Zurek et al. 1988). Tightly bound, low angular momentum material in halo centers tends to lose angular momentum and become more tightly bound, while loosely bound, high angular momentum material in the halo periphery tends to gain both energy and angular momentum. Through this process of nonviolent relaxation, galaxies which are born cold in small halos will remain cooler than the dark matter in general at any given stage of the hierarchy. We suggest this process is mainly responsible for the velocity bias. As supporting evidence, we offer the fact that a similar value for $b_{v}$ is measured for a population of collisionless test particles tagged at the centers of halos at high redshift in our dark matter-only run (the method of Carlberg 1991).

The main source of confusion in the various estimates of velocity bias is that each estimate arises from a different method of tracing galaxies in $N$-body simulations. The strength of our approach over previous experiments is that our galaxy tracer population is more physically reasonable than the single fluid (DM only) schemes employed in many of the studies quoted above. Collisionless schemes have not been shown to accurately trace galaxies through clustering (Summers et al. 1993). Such problems seriously undercut their correlation results. However, both the Carlberg et al. (1990) paper $\left(b_{v}=0.5\right)$ and the Katz et al. (1992) study $\left(b_{v}=1\right)$ employed an SPH algorithm to locate sites for galaxy formation. One difference between our work and these studies is that our mass resolution is an order of magnitude better. Another difference is in the fact that we chose to study a region of space containing a rich cluster while the other studies examined random volumes. Still, it remains unclear whether these differences could be responsible for the range of estimates in $b_{v}$.

One weakness when considering the impact on observations is that we do not evolve the system to the present day. If dynamical friction or some other systematic process is allowed to act for another 8.6 billion yr, then values of $b_{v}$ may get even smaller. However, during that period one would expect mergers and accretion to provide a fresh supply of higher velocity material to the cluster, perhaps counteracting any secular cooling. If, however, nonviolent relaxation is the principal mechanism, then we argue that the mild dependence on redshift seen in Figure 26 implies that the results at $z=0$ should not differ substantially from the results already in hand.

\section{SUMMARY AND DISCUSSION}

We have simulated the coupled evolution of baryons and dark matter in a $16 \mathrm{Mpc}$ region of a CDM universe with $10 \%$ baryon fraction. The baryons experience gravity due to both mass components and are subject to pressure forces, shock heating, and radiative cooling. No star formation mechanism is implemented. We identify a galaxy population with cold, collapsed groups of baryon particles above a fixed physical density of $\sim 0.5 \mathrm{~cm}^{-3}$. The simulation resolves $L_{*}$ galaxies with $10^{3}$ particles, an order of magnitude improvement over previous work.

The initial conditions were constrained in order to produce a modest cluster of galaxies in the region simulated. The DC density enhancement induced by the cluster prevented us from evolving to the present day; we follow the dynamics to a redshift $z=1$. Our main results are summarized as follows.

1. A multiphase structure develops in the baryons consisting of cold collapsed, hot collapsed, and cold, uncollapsed ("void") material. The collapsed fraction of $75 \%$ is consistent with Press-Schechter expectations. The partition of the collapsed fraction between hot and cold phases is sensitive to the minimum resolved mass scale. Higher resolution leads to more efficient cooling at small mass scales and early epochs (the "cooling catastrophe"). Balance between cooling and heating mechanisms such as photoionization or supernova blast waves will ultimately control these filling factors.

2. Galaxy formation in dark halos is seen to trace out the overall filamentary structure of the dark matter at high redshift, though the voids are not completely empty of galaxies. Material drains from filaments to form a central cluster. The compact nature of the dissipated globs leads to their remaining distinct, self-gravitating systems within the cluster, alleviating the overmerger problem which plagues collisionless simulations. With an effective spatial resolution of $\sim 10 \mathrm{kpc}$, the galaxy merger rates in the simulation are still likely to overestimate that of real galaxies. The fact that the two largest dark halos house very massive "central dominant" galaxies with complex merger histories is an indication that overmerging remains a problem, though at a level much reduced relative to collisionless experiments.

3. The number of resolved galaxies in the volume grows to $\sim 200$ by $z=2$ and remains steady to $z=1$. High-redshift formation of QSOs is not a major problem for biased CDM, unless the mass scale of host galaxies is much larger than $10^{11}$ $M_{\odot}$. At $z=1$, the mass function of globs has a Schechter form with "faint end" slope $\alpha_{g}=-1.39 \pm 0.12$, shallower than the halo mass function slope but steeper than the observed luminosity function. The inferred number density of galaxies with baryon mass above $10^{10} M_{\odot}$ is nearly an order of magnitude larger than the present, observed abundance of $L_{*} / 10$ galaxies. This population could represent the excess counts of faint blue galaxies, if a suitably complex star formation history is invoked.

4. The merger rate of galaxies was found to be lower than that of dark halos by about a factor 2 . Over the final $\frac{1}{3}$ of the simulation, $45 \%$ of the halos suffered mergers involving progenitors with mass ratio larger than 0.1 , while the equivalent rate for globs was $23 \%$. The $\sim 10 \mathrm{kpc}$ minimum spatial resolution and lack of a collisionless "star" component in our experiment imply that the true galaxy merger rate may be even smaller. It remains to be seen whether rates as low as those argued for by Tóth \& Ostriker can be reached.

5. We confirm galactic disk formation as a generic outcome of hierarchical clustering. This is the first experiment to grow a population of centrifugally supported disks in a natural, largescale setting. Examination of the Tully-Fisher relation, phrased in terms of baryon mass and rotation velocity, finds good agreement with a power law $M_{B} \propto v_{\text {rot }}^{\alpha}$ with $\alpha \simeq 2.5$ is the slope after correcting for numerical resolution effects. The scatter in this relation is extremely tight; the rms residual in $\log \left(M_{B}\right)$ is 0.05 , equivalent to $0.13 \mathrm{mag}$. Reconciling the slope with observations requires that the star formation efficiency vary systematically with mass so that $L \propto M_{B}^{\beta / 2.5}$, where $\beta \simeq 4$ is the observed Tully-Fisher slope. This relation would also flatten the faint end slope of the inferred luminosity function to a value $\alpha=-1.24$, in better agreement with observations.

6. The glob population at $z=3$ is spatially biased with respect to the dark matter; a linear bias $b=2$ is found from both the correlation function and a counts-in-cells analysis, with more massive objects being more strongly biased. At $z=1$, after collapse of the central cluster, the positive spatial bias is eliminated for all but the most massive globs. A counts- 
in-cells analysis reveals a complex, nonlinear relation between the mass and galaxy overdensities. The highest density cell shows a spatial antibias, i.e., the density contrast in galaxy number is less than the mass density contrast of the dark matter. This arises from merging of globs in the cluster environment. We suspect a more refined treatment would reduce the amount of merging, thereby preserving some amount of spatial bias at late epochs.

7. Analysis of the one-dimensional, pairwise peculiar velocities of the globs and dark matter indicates that galaxies are "cooler" on small and intermediate scales than the dark matter. The "velocity bias" measured between 0.1 and $4 \mathrm{Mpc}$ is well approximated by $b_{v}(r)=0.7(r / 1 \mathrm{Mpc})^{0.2}$. The fact that the effect is independent of both redshift and glob mass downplays the significance of dynamical friction as the underlying mechanism. We suspect that nonviolent relaxation plays the dominant role; galaxies are born at local binding energy maxima and this trait is inherited through hierarchical clustering.

Biasing remains a mysterious and contentious issue. Previous attacks on the problem with different algorithms seem to have generated as much heat as light. The issue may remain undecided until a generation of experiments emerges capable of handling star formation and feedback with $1 \mathrm{kpc}$ resolution in greater than $10 \mathrm{Mpc}$ regions. Our best estimate is that galaxies are probably mildly biased in both real and velocity space. With the COBE DMR measurement providing a spectrum normalization on large scales, the degree and character of biasing plays a limiting role in attempts to place robust constraints on the fluctuation spectrum on intermediate and small scales. It is imperative that more realistic numerical models addressing this question be pursued.

What are the ramifications of this work for the popular cold dark matter model? With our chosen "standard" normalization, which is just outside of the $2 \sigma C O B E$ error bars, galaxy formation "begins" at rather high redshifts $z \simeq 6$ and the number of galaxies grows until $z=2$, flattening thereafter at a level several times above the local abundance of bright galaxies. The abundance of systems at high $z$ is beneficial from the point of view of forming QSOs and, perhaps, faint blue galaxies. However, the star formation history in these objects must be such that the majority of the low-mass systems are undetected in local surveys. It remains to be seen whether the overabundance of low-mass galaxies presents a serious threat to the CDM model.

Small-scale peculiar velocities apparently remain problem- atic for CDM. The velocity bias of 0.7 at $1 \mathrm{Mpc}$ seen in our experiment is insufficient to bring pairwise peculiar velocities down to observed levels. The large effect seen in the Couchman $\&$ Carlberg (1992) simulation resulted from a poor choice of galaxy tracer algorithm (Gelb \& Bertschinger 1993; Summers et al. 1993). We feel that the most sensible algorithms to date have yielded values $\gtrsim 0.7$ at $1 \mathrm{Mpc}$, and this number represents a conservative lower bound on the amplitude of $b_{v}$.

Modifications to the assumed cosmology could alleviate some of these problems. For example, a mixed (hot + cold) dark matter model can solve the small-scale velocity problem (Davis, Summers, \& Schlegel 1992) while at the same time allowing early formation of quasars (Klypin et al. 1992). In general, reducing the amount of matter clustered on small scales will lower small-scale velocities. In this regard, a low $\Omega$ CDM model with nonzero cosmological constant would fare well (Martel 1991). The reduction of the galaxy merger rate is likely to be unaffected by the assumed cosmology, since it arises from dissipational physics generic to all hierarchical clustering models. However, the overall frequency of merging is controlled by the assumed shape of the initial fluctuation spectrum and $\Omega_{0}$ (Kauffmann \& White 1993; Lacey \& Cole 1993).

Finally, the faint end slope of the luminosity function and the Tully-Fisher slope both require that the specific luminosity increase as a mild power of baryon mass. This prediction is difficult to test observationally, since it requires accounting for all the baryons associated with a galaxy, including material ejected via winds or locked up in low-mass stars. The ratio of disk to halo mass within an isophotal radius increases systematically with luminosity in observed spirals (Ashman, Persic, \& Salucci 1993), and this trend may be indicative of the required $L / M_{B}$ dependence.

We thank many colleagues for valuable discussions during the course of this project; in particular, J. Ostriker, G. Efstathiou, L. Hernquist, N. Katz, C. Frenk, and S. White. Special thanks to D. Weinberg for commas, quickness, and insight as second referee. This research was supported by NSF, in part through allocation of CPU time on a CRAY Y-MP at the San Diego Supercomputer Center. We commend the staff of SDSC for their excellent system organization and management. Support was also received from NASA, in part through Theory grant NAGW-2367. A. E. E. acknowledges support from the Miller Institute for Basic Research in Science during the embryonic stages of this project.

\section{REFERENCES}

Arnaud, M., Rothenflug, R., Boulade, O., Vigroux, L., \& Vangioni-Flam, E. 1991, A\&A, 254, 49

Ashman, K. M., Salucci, P., \& Persic, M. 1993, ApJ, in press

Bardeen, J. M., Bond, J. R., Kaiser, N., \& Szalay, A. S. 1986, ApJ, 304, 15

Barnes, J. 1989, Nature, 338, 123

Beacom, J. F., Dominik, K. G., Melott, A. L., Perkins, S. P., \& Shandarin, S. F. 1991, ApJ, 372, 351

Bean, A. J., Efstathiou, G., Ellis, R. S., Peterson, B. A., \& Shanks, T. 1983, MNRAS, 205, 605

Bertschinger, E. 1987, ApJ, 323, L103

Bingelli, B., Sandage, A., \& Tammann, G. A. 1985, AJ, 90, 1681

Blanchard, A., Valls-Gabaud, D., \& Mamon, G. A. 1992, A\&A, 264, 365

Bothun, G. D., Impey, C. D., \& Malin, D. F. 1991, ApJ, 376, 404

Broadhurst, T. J., Ellis, R. S., \& Shanks, T. 1988, MNRAS, 235, 827

Carignan, C., \& Freeman, K. C. 1988, ApJ, 332, L33

Carlberg, R. G. 1988, ApJ, 324, 664

-1990, ApJ, 359, L1

1991, ApJ, 367, 385

Carlberg, R. G., \& Couchman, H. M. P. 1989, ApJ, 340, 47

Carlberg, R. G., Couchman, H. M. P., \& Thomas, P. A. 1990, 352, L29

Carlberg, R. G., \& Dubinski, J. 1991, ApJ, 369, 13

Casertano, S., \& Hut, P. 1985, ApJ, 298, 80

Cavaliere, A., Colafrancesco, S., \& Scaramella, R. 1991, ApJ, 380, 15

Cen, R., Jameson, A., Liu, F., \& Ostriker, J. 1990, ApJ, 362, L41

Cen, R., \& Ostriker, J. 1992a, ApJ, 393, 22 (C\&O) 1992b, ApJ, 399, L113

Chiang, W. H., Ryu, D., \& Vishniac, E. T. 1989, ApJ, 339, 603

Cole, S. 1991, ApJ, 367, 45

Cole, S., \& Kaiser, N. 1989, MNRAS, 237, 1127

Colless, M. M., Ellis, R. S., Taylor, K., \& Hook, R. N. 1989, MNRAS, 244, 408

Couchman, H. M. P., \& Carlberg, R. G. 1992, ApJ, 389, 453

David, L. P., Arnaud, K. A., Forman, W., \& Jones, C. 1990, ApJ, 356, 72

Davis, M., Efstathiou, G., Frenk, C. S., \& White, S. D. M. 1985, ApJ, 292, 371

Davis, M., \& Peebles, P. J. E. 1983, ApJ, 267, 465

Davis, M., Summers, F. J., \& Schlegel, D. 1992, Nature, 359, 353

Dekel, A., \& Silk, J. 1986, ApJ, 303, 39

Djorgovski, S., De Carvalho, R., \& Han, M.-S. 1988, in Extragalactic Distance

Scale, ed. S. van den Bergh (ASP Conf. Ser., 4), 203

Dressler, A. 1980, ApJ, 236, 351

Dubinski, J., \& Carlberg, R. 1991, ApJ, 378, 496 


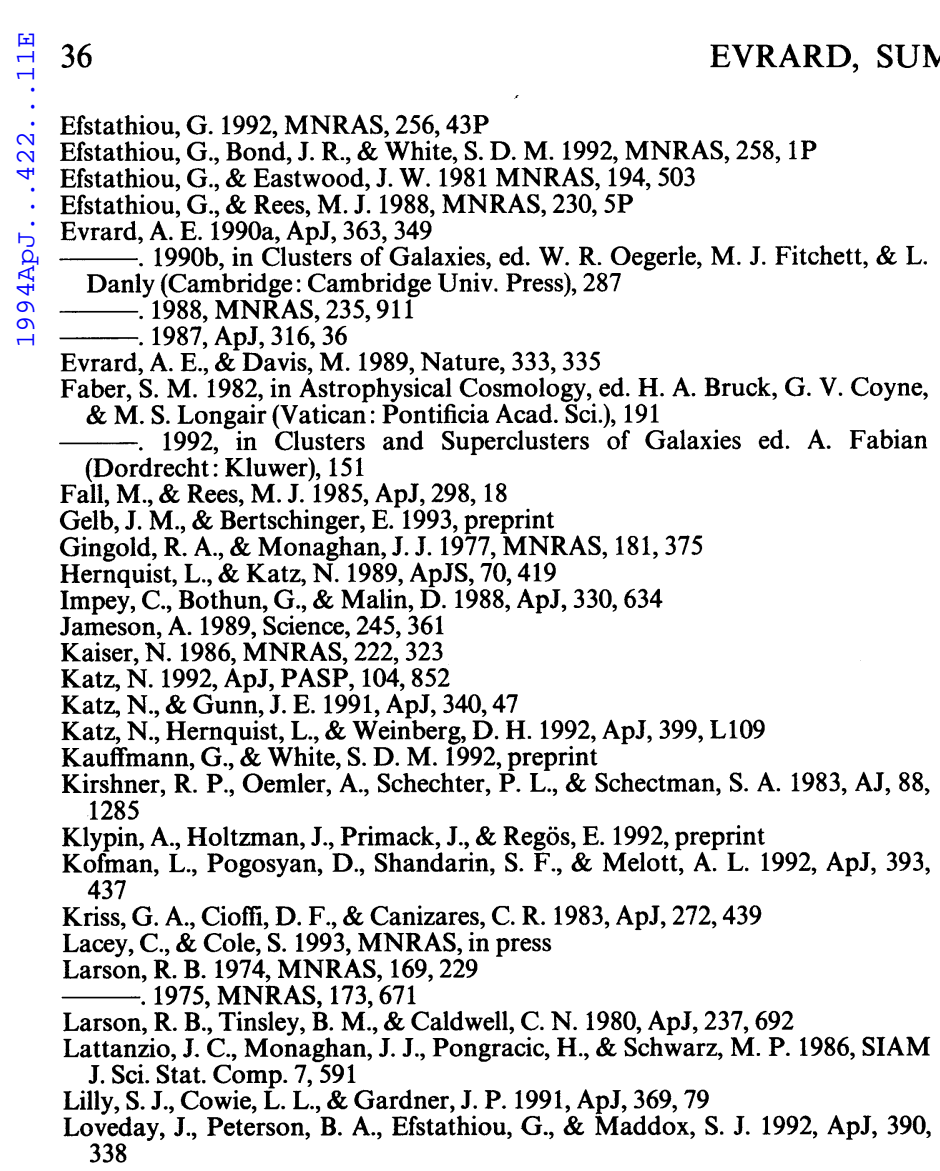

Loveday, J., Peterson, B. A., Efstathiou, G., \& Maddox, S. J. 1992, ApJ, 390, 338

Lucy, L. B. 1977, AJ, 82, 1013

Maddox, S. J., Sutherland, W. J., Efstathiou, G., Peterson, B. A., \& Loveday, J. 1990, MNRAS, 247, $1 \mathrm{P}$

Martel, H. 1991, ApJ, 367, 7

Miralda-Escudé, J., \& Ostriker, J. P. 1990, ApJ, 350, 1

Monaghan, J. J. 1992, ARA\&A, 30, 394

Navarro, J. F., \& Benz, W. 1991, ApJ, 380, 320

Navarro, J. F., \& White, S. D. M. 1993, preprin

Peebles, P. J. E. 1980, The Large-Scale Structure of the Universe (Princeton: Princeton Univ. Press)

Persic, M., \& Salucci, P. 1988, MNRAS, 234, 131 1992, MNRAS, 258, 14P

Phillipps, S., \& Disney, M. 1985, A\&A, 148, 234

Postman, M., \& Geller, M. J. 1984, ApJ, 281, 95

Press, W. H., \& Schechter, P. 1974, ApJ, 187, 425

Rees, M. J., \& Ostriker, J. P. 1977, ApJ, 179, 541

Rubin, V., et al. 1982, ApJ, 289, 81

Ryu, D., Vishniac, E. T., \& Chiang, W. H. 1990, ApJ, 354, 389

Sandage, A., Tammann, G. A., \& Yahil, A. 1979, ApJ, 232, 352

Sarazin, C. L. 1986, Rev. Mod. Phys., 58, 1

Shanks, T., Fong, R., Boyle, B. J., \& Peterson, B. A. 1989, in The Epoch of Galaxy Formation, ed. C. S. Frenk et al. (Dordrecht: Kluwer), 141

Silk, J. I. 1977, ApJ, 211, 638

Smoot, G. F., et al. 1992, ApJ, 396, L1

Summers, F. J., Davis, M., \& Evrard, A. E. 1993, in preparation

Thomas, P. T., \& Couchman, H. M. P. 1992, MNRAS, 257, 11

Tóth, G., \& Ostriker, J. P. 1992, ApJ, 389, 5

Turner, E. L. 1991, AJ, 101, 5

Umemeura, M. 1992, preprint

White, S. D. M., Davis, M., Efstathiou, G., \& Frenk, C. S. 1987, Nature, 330 451

White, S. D. M., Efstathiou, G., \& Frenk, C. S. 1993, MNRAS, 262, 1023

White, S. D. M., \& Frenk, C. S. 1991, ApJ, 389, 52

White, S. D. M., \& Rees, M. J. 1978, MNRAS, 183, 341

Whitmore, B. C. 1990, in Clusters of Galaxies, ed. W. R. Oegerle, M. J. Fitchett \& L. Danly (Cambridge: Cambridge Univ. Press), 139

Yuan, W., Centrella, J. M., \& Norman, M. L. 1991, ApJ, 376, L29

Zurek, W., Quinn, P., \& Salmon, J. 1988, ApJ, 330, 519 